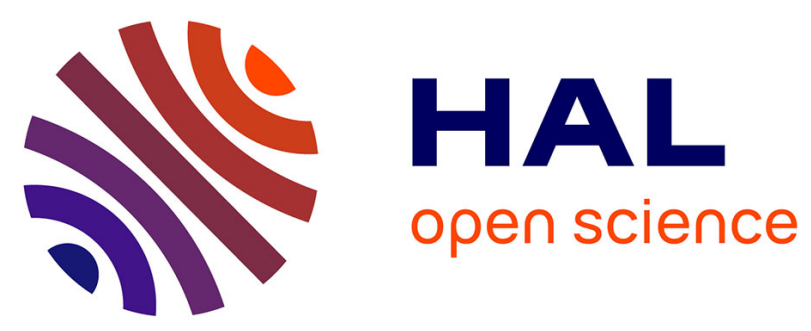

\title{
Évolutions paysagères et occupations humaines passées du Marais poitevin occidental durant la fin de l'Holocène
}

Pierre Pouzet, Mohamed Maanan, Sabine Schmidt, Gaëlle Dieulefet, Jean-Marc Large, Marc Robin

\section{- To cite this version:}

Pierre Pouzet, Mohamed Maanan, Sabine Schmidt, Gaëlle Dieulefet, Jean-Marc Large, et al.. Évolutions paysagères et occupations humaines passées du Marais poitevin occidental durant la fin de l'Holocène. Géomorphologie: relief, processus, environnement, 2021, 27 (4), pp.263-278. 10.4000/geomorphologie.16139 . hal-03507163

\section{HAL Id: hal-03507163 \\ https://hal.science/hal-03507163}

Submitted on 20 Jan 2022

HAL is a multi-disciplinary open access archive for the deposit and dissemination of scientific research documents, whether they are published or not. The documents may come from teaching and research institutions in France or abroad, or from public or private research centers.
L'archive ouverte pluridisciplinaire HAL, est destinée au dépôt et à la diffusion de documents scientifiques de niveau recherche, publiés ou non, émanant des établissements d'enseignement et de recherche français ou étrangers, des laboratoires publics ou privés. 


\title{
Évolutions paysagères et occupations humaines passées du Marais poitevin occidental durant la fin de l'Holocène
}

\section{Late Holocene landscape evolutions and ancient human occupations of the Western Marais Poitevin}

\author{
Pierre Pouzet ${ }^{\mathrm{a}^{*}}$, Mohamed Maanan ${ }^{\mathrm{b}}$, Sabine Schmidt ${ }^{\mathrm{c}}$, Gaëlle Dieulefet ${ }^{\mathrm{d}}$, Jean-Marc Large ${ }^{\mathrm{e}}$, Marc Robin ${ }^{\mathrm{b}}$ \\ a Université de Nantes, UMS CNRS 3281 OSUNA - OR2C, chemin de la Censive du Tertre, 44300 Nantes, France. \\ ${ }^{\mathrm{b}}$ Université de Nantes, UMR CNRS 6554 LETG, chemin de la Censive du Tertre, 44300 Nantes, France. \\ ' Université de Bordeaux, UMR CNRS 5805 EPOC, EPHE, Allée Geoffroy Saint-Hilaire 33615 Pessac, France. \\ 'Université de Nantes, UMR CNRS 6566 CReAAH - LARA, Chemin de la Censive-du-Tertre 44300 Nantes, France. \\ ' UMR CNRS 6566 CReAAH ; 25, rue Saint-Vincent-de-Paul, 85000 La Roche-sur-Yon, France.
}

INFORMATION SUR L'ARTICLE

Reçu le 2 avril 2021

Reçu sous sa forme révisée le 28 octobre 2021

Définitivement accepté le 29 octobre 2021

*Auteur correspondant. Tél : +33 (0)2 51125314 Courriels : pierre.pouzet@univ-nantes.fr (P. Pouzet) mohamed.maanan@univ-nantes.fr (M. Maanan) sabine.schmidt@u-bordeaux.fr (S. Schmidt) gaelle.dieulefet@univ-nantes.fr (G. Dieulefet) jlarge2@wanadoo.fr (J. M. Large) marc.robin@univ-nantes.fr (M. Robin)

\begin{abstract}
RÉSUMÉ
Dans le contexte actuel du changement climatique, l'analyse croisée de lévolution paléoenvironnementale et paléosociétale d'un secteur littoral vulnérable illustre différentes formes doccupations humaines liées à leur environnement durant les derniers millénaires. Une analyse sédimentologique couplée avec des cartographies historiques permet de reconstruire et de dater finement lévolution des principaux paléopaysages de la bordure ouest du Marais poitevin. Cette analyse confirme que cet espace a profondément évolué, notamment durant l'Antiquité et le début du Moyen Âge, périodes durant lesquelles l'ancien golfe maritime s'est transformé en un marais aujourd'hui colmaté. En parallèle, l'évolution de l’occupation humaine historique est qualifiée par des données géoarchéologiques. Des vestiges d'anciennes pêcheries et empierrements ont été identifiés à proximité des points rocheux. Ils témoignent d'une occupation humaine historique dans les anciennes îles, au sein desquelles les Hommes se trouvaient en sécurité face aux aléas météo-marins.
\end{abstract}

Mots clés : paléoenvironnement, sédimentologie, cartographie ancienne, Marais poitevin, géoarchéologie, occupation littorale

\section{ABSTRACT}

In the current context of climate change, crossing analyses of the palaeoenvironmental and paleosocietal evolution of a vulnerable coastal area illustrates the different forms of human occupations that have been linked to their environment during the last millenaries. A sedimentological analysis combined with historical maps allows us to rebuild and date precisely the evolution of the major paleolandscapes of the Marais Poitevin western border. This analysis confirms that this territory has deeply changed, especially during Antiquity and the early-Middle Ages, when the ancient maritime gulf turned into a marsh that is nowadays infilled. The evolution of historical human occupation is simultaneously qualified thanks to geoarcheological data. Remains of old fisheries and enstonements were identified next to rocky spots. They testify about an historical human occupation of the former islands, where people were protected from coastal hazards.

Keywords: palaeoenvironment, sedimentology, ancient cartography, Marais Poitevin, geoarcheology, coastal occupation

\section{Introduction}

Dans le contexte actuel du changement climatique et de l'élévation du niveau marin, l'analyse de l'évolution paysagère passée de littoraux vulnérables est essentielle. Elle permet de comprendre l'occupation humaine historique des sociétés face aux grandes mutations environnementales qui se sont produites au cours des derniers siècles et/ou millénaires. Cette étude prend l'exemple d'un environnement très sensible, récemment impacté par la submersion marine provoquée par la tempête Xynthia en février 2010 (Mercier, 2019). La lagune de la Belle-Henriette est une zone humide localisée entre les communes de La-Faute-sur-Mer et de La-Tranche-surMer dans la bordure ouest du Marais poitevin. Elle s'est formée récemment, il y a près d'un siècle selon les travaux de Bouhier
(Bouhier, 1957). Les zones humides font office de tampon pour les espaces situés en arrière (Verger, 2009). Pourtant, leur vulnérabilité est très importante. L'événement Xynthia a durement touché la région, produisant de nombreuses brèches dans les cordons dunaires et dans les digues, submergeant ainsi des espaces habités (Chauveau et al., 2011).

Pour comprendre les évolutions croisées de l'environnement et de l'adaptation des sociétés littorales, des archives sédimentologiques datées permettent dans un premier temps une reconstruction fine de la lagune de la Belle-Henriette et du colmatage de la partie ouest $\mathrm{du}$ Marais poitevin. Ces hypothèses paléoenvironnementales sont croisées avec une reconstruction du paysage parl'étude de documents cartographiques anciens. Enfin, l'étude des cartes historiques est également croisée avec l'analyse de vestiges archéologiques pour 
appréhender les mutations spatiales des sociétés littorales par une approche qualitative. En couplant la sédimentologie avec des cartographies historiques, ainsi que des analyses géoarchéologiques, nous tentons de comprendre comment l'Homme a su s'adapter face à son environnement au cours des derniers millénaires. L'analyse croisée des évolutions paléoenvironnementale et paléosociétale de ce secteur vulnérable nous permet d'offrir quelques clés de compréhension sur ces coévolutions.

\section{Outils et méthodes}

\subsection{Zone d'étude}

Le Marais poitevin est un espace très sensible et d'un grand intérêt écologique, disposant d'une biodiversité très riche, à l'image de nombreuses zones humides mondiales (Wolanski et al., 2009). Cet espace, seconde plus vaste zone humide de France, a pourtant profondément muté au cours des derniers siècles. Le Marais poitevin est un ancien golfe où débouchaient notamment les fleuves de la Sèvre Niortaise et l'actuel Lay, anciennement appelé Saint Benoist. Cet ancien golfe a été comblé par des alluvions quaternaires marines et fluviatiles, avec comme principale formation une argile appelée "bri" (Welsch, 1916). Le marais est aujourd'hui divisé en deux parties distinctes : les marais mouillés à l'est dans le fond de l'ancien golfe correspondent aux lits majeurs inondables des différents cours d'eau, divisés par des fossés et rigoles ; et les marais desséchés à l'ouest, larges étendues de verdure parsemées d'anciens marais salants et surplombées par les anciennes îles calcaires, à l'abri des inondations fluviales (Jambu et Nijs, 1966). Les poldérisations y ont été massives depuis le XVII siècle, couvrant aujourd'hui près de $75 \mathrm{~km}^{2}$, concordant avec la disparition de surfaces de schorres (Godet et al., 2015). Plus récemment, les prairies ont été reconverties en cultures au cours de la seconde moitié du $\mathrm{XX}^{\mathrm{e}}$ siècle (Godet et Thomas, 2013), et une forte littoralisation (une concentration des populations et des activités humaines le long des littoraux selon Géoconfluences) y a été observée le long du trait de côte (Pouzet et al., 2015).

Sur la bordure occidentale de ce marais le long de l'océan Atlantique, la lagune de la Belle-Henriette est une lagune récente de quatre kilomètres de long couvrant près de 200 hectares dans un environnement très dynamique (fig. 1). Nous avons choisi de carotter précisément l'anse des Roullières, car c'est la partie la plus ancienne de la lagune selon les cartographies historiques, et donc le site le plus pertinent pour discuter de son évolution paléoenvironnementale. Cette anse est composée d'une vasière colonisée par une végétation de schorre située entre 0 et $2 \mathrm{~m}$ NGF selon les données issues de campagnes aéroportées topographiques du Lidar datant de 2020 à La-Faute-sur-Mer, financées

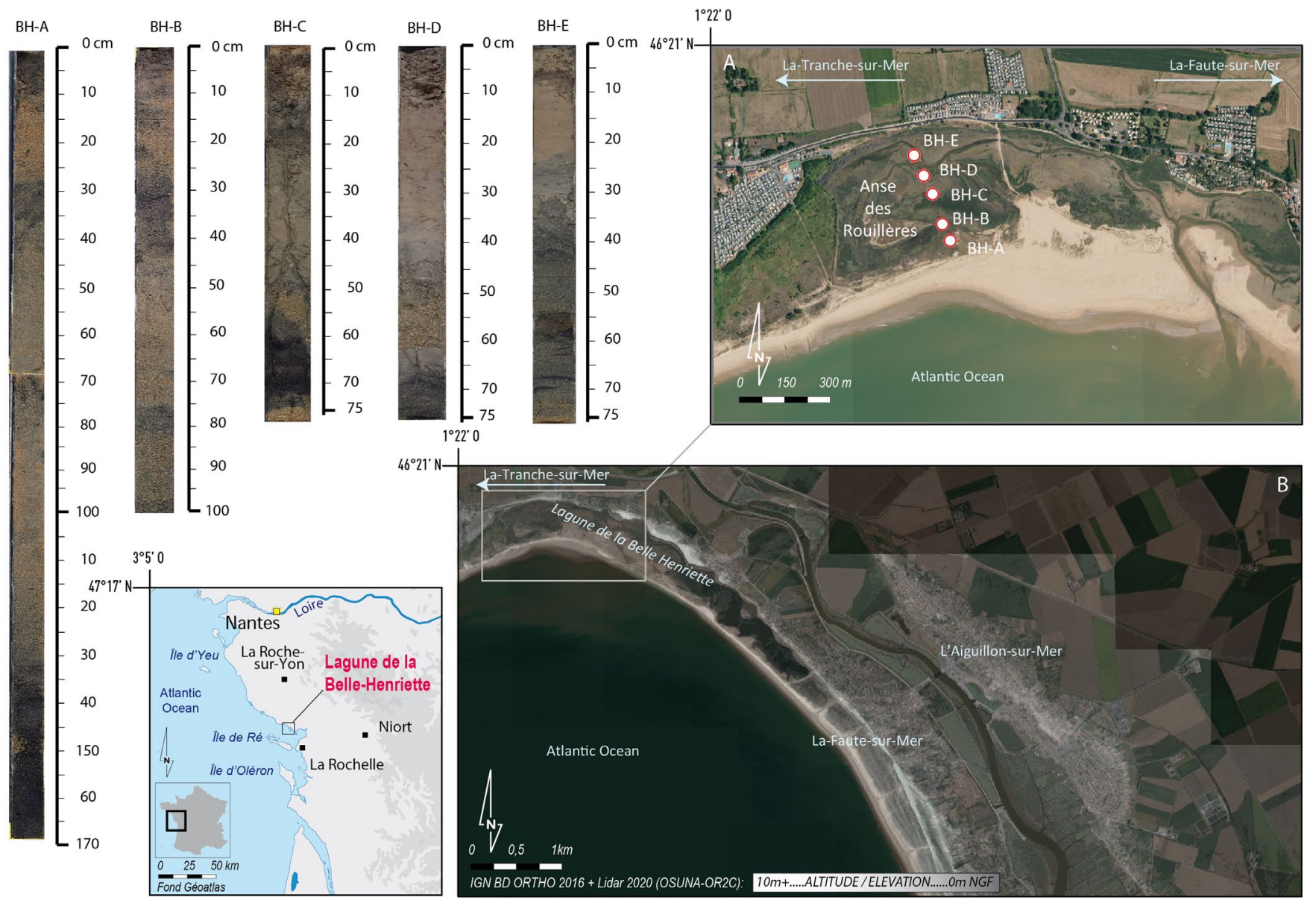

Fig. 1 - Présentation de la zone d'étude.

A : Localisation des carottes prélevées dans l'Anse des Rouillères; B : Topographie de la lagune de la Belle-Henriette.

Fig. 1 - Presentation of the study area.

A: Location of the cores extracted from the Anse des Rouilleres; B: Topography of the Belle-Henriette lagoon. 
par l'Observatoire Régional des Risques Côtiers, OR2C (Kerguillec et al., 2019). La lagune est bordée de part et d'autre de deux cordons dunaires culminant à près de $10 \mathrm{~m}$ NGF, l'isolant de l'océan à son sudouest et des marais anthropisés et de l'estuaire du Lay à son nord-est (fig. 1). De nombreuses études récentes ont démontré que ce littoral est soumis à de puissantes dynamiques morphogéniques (Chaumillon et al., 2004 ; Chaumillon et Weber, 2006 ; Allard et al., 2008 ; Poirier et al., 2017). Sur la côte atlantique, l'élévation du niveau de la mer a été ralentie vers $7000 \mathrm{BP}$. Dans cette région, un point d'inflexion notable est observé dans les courbes de remontée du niveau de la mer entre 7000 et $6000 \mathrm{BP}(2,6 \mathrm{~mm} /$ an d'élévation du niveau de la mer avant et $0,8 \mathrm{~mm} /$ an après). Cette inflexion témoigne d'un affaiblissement de l'élévation du niveau marin, entrainant alors une stabilisation relative du littoral (Stéphan et Goslin, 2014 ; Garcia-Artola et al., 2018, fig. 3).

\subsection{Sédimentologie}

\subsubsection{Prélèvements sédimentologiques}

Cinq carottes de sédiments ont été prélevées durant deux campagnes de terrain réalisées en octobre 2018 et 2019, suivant un transect allant du sud-est vers le nord-ouest (fig. 1). La première campagne datant d'octobre 2018 a permis le prélèvement de deux carottes BH-A et $\mathrm{BH}-\mathrm{B}$ sur le cordon dunaire protecteur séparant la lagune de l'océan. Un carottier motorisé à percussion "vibracore " Eijkelkamp ${ }^{\odot}$ a été utilisé pour carotter ces sols compacts (Pouzet et Maanan, 2020b). La carotte BH-A a une longueur de $170 \mathrm{~cm}$ et la carotte BH-B de $100 \mathrm{~cm}$. La seconde campagne réalisée en octobre 2019 a permis le prélèvement de trois carottes extraites au cœur de la lagune (BH-C, BH-D et BH-E), à l'aide d'un carottier gravitaire "Beeker " Eijkelkamp ${ }^{\odot}$, efficace pour échantillonner dans un sol argileux ou limoneux humide (Pouzet et al., 2019). Les carottes BH-C, BH-D et BH-E ont toutes des longueurs d'environ $76 \mathrm{~cm}$.

\subsubsection{Analyses en laboratoire}

Les carottes ont été ouvertes longitudinalement et une photographie a été prise dès l'ouverture pour préserver les couleurs des sédiments (Pouzet et Maanan, 2020a). Un log stratigraphique a ensuite été construit pour décrire les différents faciès observés. Des prélèvements ont été réalisés tous les centimètres dans les cinq carottes pour mesurer finement la granulométrie de l'ensemble des faciès au Malvern Mastersizer Hydro $2000 G^{\odot}$. Les sédiments sont au préalable placés dans une solution comprenant de l'eau distillée et de l'Héxamétaphosphate de sodium (Na6O18P6) à $5 \%$, pendant 48h (Gee et Or, 2002 ; Kilmer et Alexander, 1949). Le logiciel GRADISTAT V8 ${ }^{\oplus}$ a permis de transformer la distribution granulométrique brute en une distribution ordonnée selon des classes de tailles de grains (argiles, limons, sables, éléments grossiers) (Blott et Pye, 2001). La méthode de Folk et Ward a été retenue pour le calcul des différents indicateurs statistiques (Folk et Ward, 1957).

\subsubsection{Datation}

Les modèles d'âge des carottes BH-B, BH-C, BH-D et BH-E ont été établis à partir de la combinaison de deux radionucléides adaptés aux échelles de temps pluridécennales. D’une part, le ${ }^{210} \mathrm{~Pb}\left(\mathrm{t}_{1 / 2}=22,2\right.$ ans $)$ est un radio-isotope naturel dont les apports atmosphériques s'incorporent rapidement dans le sédiment. $\mathrm{Ce}{ }^{210} \mathrm{~Pb}$ dit en excès décroît avec la profondeur dans les sédiments en fonction de sa période radioactive et de la vitesse de sédimentation. Les profils de ${ }^{210} \mathrm{~Pb}_{\mathrm{xs}}$ dans les sédiments permettent d'estimer des taux de sédimentation et ainsi de dater les sédiments. Ces estimations peuvent être comparées avec celles dérivées du radio-isotope artificiel ${ }^{137} \mathrm{Cs}\left(\mathrm{t}_{1 / 2}=30 \mathrm{ans}\right)$. Deux pics liés aux essais nucléaires atmosphériques (début en 1950 avec un maximum des retombées en 1963 pour l'hémisphère nord), et à l'accident de Tchernobyl (1986) sont ainsi identifiés.

La détermination des activités des radioéléments d'intérêt $\left({ }^{210} \mathrm{~Pb},{ }^{226} \mathrm{Ra},{ }^{137} \mathrm{Cs}\right)$ repose sur la mesure non destructive par spectrométrie gamma directe d'environ $2 \mathrm{~g}$ de sédiments secs. Le détecteur utilisé est un spectromètre gamma à bas bruit de fond de type Broad Energy (BeGE ${ }^{\mathrm{TM}}$, Mirion Technologies). Les taux d'accumulation sédimentaires ont été calculés à partir des profils de ${ }^{210} \mathrm{~Pb}_{\mathrm{xs}}$ selon la profondeur des échantillons. L’année de dépôt de chaque couche de sédiments a ensuite été estimée en considérant que l'âge du sommet de la carotte correspondait à la date d'échantillonnage (2018 pour BH-A et BH-B et 2019 pour BH-C, BH-D et BH-E) selon la méthode Constant Flux, Constant Sedimentation Rate (CFCS).

Enfin, pour estimer les âges des horizons les plus profonds où les activités $\mathrm{du}^{210} \mathrm{~Pb}$ ne sont plus détectables, une coquille marine a été prélevée dans la carotte $\mathrm{BH}-\mathrm{C}$ et a été datée au radiocarbone $\left({ }^{14} \mathrm{C}\right)$. Cette coquille permet la calibration des horizons sableux situés entre 50 et $80 \mathrm{~cm}$ de profondeur des trois carottes lagunaires $\mathrm{BH}-\mathrm{C}, \mathrm{BH}-\mathrm{D}$ et $\mathrm{BH}-\mathrm{E}$ qui ont été prélevées dans un environnement commun. L'élément extrait est une coquille de Coque commune Cerastoderma edule identifiée au centimètre 62 de la carotte $\mathrm{BH}-\mathrm{C}$, datée à $1810 \pm 30$ ans par Spectrométrie de masse par accélérateur (AMS) (Zoppi, 2010). Cette analyse a été réalisée par le laboratoire BETA Analytic, qui a corrigé la datation à l'aide des deux courbes atmosphérique (IntCal13) et marine (Marine13) selon la référence de Reimer (Reimer et al., 2013) ; ainsi que de la Marine Correction Database selon les travaux de Tisnérat-Laborde (Tisnérat-Laborde et al., 2010).

\subsection{Létude de données spatialisées historiques et géoarchéologiques}

Dans un premier temps, pour étudier les formes d'occupations humaines plus anciennes, une reprise de la documentation a été réalisée à partir des publications scientifiques, des bases de données nationales et des rapports d'opérations. Les opérations préventives et programmées apportent les informations de premières mains les plus récentes. Ces données archéologiques croisées aux données géographiques offrent un regard complet sur la présence de vestiges et leur inscription dans le territoire actuel au sein d'une approche géoarchéologique.

Une étude diachronique basée sur des cartographies et photographies aériennes anciennes a également été menée pour appréhender ces variations passées. Cinquante-cinq cartes anciennes présentant l'évolution de la partie ouest du Marais poitevin ont été extraites des archives de la BNF Gallica, et des archives départementales de Vendée (85) et de Charente- 
Maritime (17). Les cartes de Claude Masse, stockées au Service Historique de la Défense (antenne de Rochefort), ont également été étudiées. En parallèle, la base de données IGN (Institut national de l'information géographique et forestière) Remonter le Temps (https://remonterletemps.ign.fr/) nous permet d'avoir accès aux cartes de Cassini (1740), de l'État-major (1820) et des photographies aériennes prises depuis 1945. Cette date marque le début des campagnes de photographies aériennes prises à la BelleHenriette. Cependant, après consultation des fonds de Fernand Verger, il est apparu que des campagnes de photographies plus anciennes sont également disponibles depuis 1924. Enfin, la morphologie récente de la lagune est appréhendée par la $\mathrm{BD}$ ORTHO de l'IGN datant de 2016, croisées avec les données issues de campagnes aéroportées topographiques du Lidar datant de 2020 à La-Faute-sur-Mer (Kerguillec et al., 2019).

\section{Résultats}
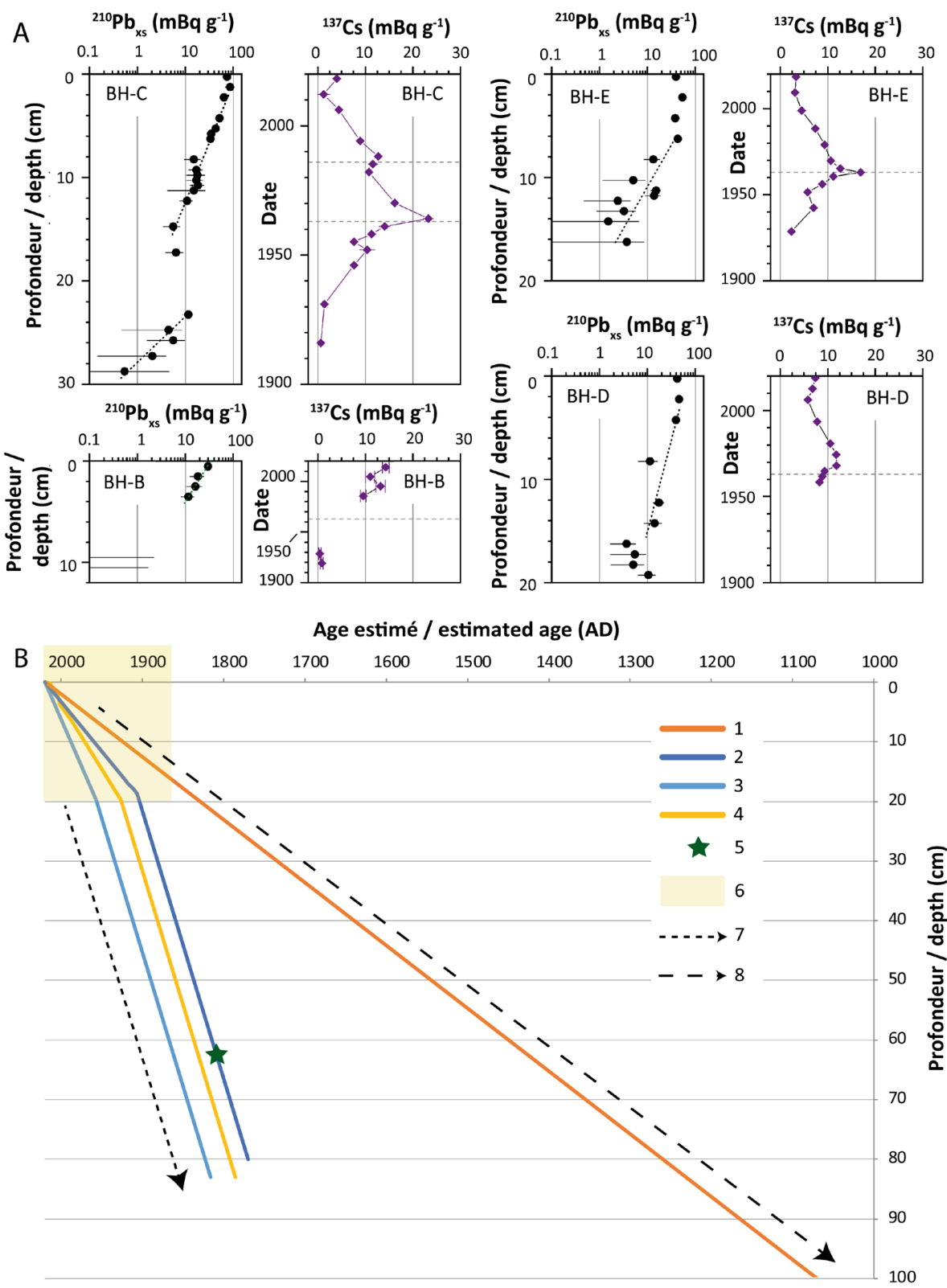

\subsection{Datations et création du modèle d'âge}

\subsubsection{Datations des carottes $B H-C, B H-D$, et $B H-E$}

Les trois carottes $\mathrm{BH}-\mathrm{C}, \mathrm{BH}-\mathrm{D}$ et $\mathrm{BH}-\mathrm{E}$ prélevées au cœur de l'anse des Rouillères (fig. 1) présentent un faciès superficiel limoneux d'environ $16 \mathrm{~cm}$, ce qui a permis d'établir des profils détaillés $\mathrm{du}^{210} \mathrm{~Pb}_{\mathrm{xs}}$ et de ${ }^{137} \mathrm{Cs}$. Des activités significatives de ${ }^{210} \mathrm{~Pb}_{\mathrm{xs}}$ ont été mesurées jusqu'à $30 \mathrm{~cm}$ selon les sites, correspondant à des taux d'accumulation sédimentaire du même ordre de grandeur : $0,17 \mathrm{~cm} / \mathrm{an}$ pour la carotte $\mathrm{BH}-\mathrm{C}, 0,32 \mathrm{~cm} / \mathrm{an}$ pour $\mathrm{BH}-\mathrm{D}$ et $0,215 \mathrm{~cm} / \mathrm{an}$ pour BH-E (fig. 2). Ces analyses nous ont permis d'établir une datation pour les premiers décimètres de ces carottes, en assumant que l'âge du sommet des carottes est 2019, l'année de carottage. La datation basée sur le ${ }^{210} \mathrm{~Pb}_{\mathrm{xs}}$ est validée par les profils de ${ }^{137} \mathrm{Cs}$. En effet l'application de l'échelle d'âge aux données de ${ }^{137} \mathrm{Cs}$ montre que cet élément présente le pic
Fig. 2 - Résultats des datations.

$\mathrm{A}$ : Profils de ${ }^{210} \mathrm{~Pb}_{\mathrm{xs}}$ en fonction de la profondeur dans les carottes BH-B, -C, -D, et -E. Les profils de ${ }^{137} \mathrm{Cs}$ sont tracés en fonction du modèle d'âge dérivé des profils de ${ }^{210} \mathrm{~Pb}$ de chaque carotte pour tester sa validité. Les traits soulignent l'année 1963 pour toutes les carottes, et également 1986 pour la carotte BH-C. B : Modèles d'âge des carottes : 1 . BH-A et BH-B ; 2 . BH-C ; 3. BH-D ; 4. BH-E. ; 5. Coquille datée au ${ }^{14} \mathrm{C}(\mathrm{cm} .62-1810 \mathrm{AD}) ; 6$. Datation au ${ }^{210} \mathrm{~Pb} /{ }^{137} \mathrm{Cs}$; 7. Extrapolation du taux de sédimentation évalué entre la fin de la datation au ${ }^{210} \mathrm{~Pb} /{ }^{137} \mathrm{Cs}$ et la coquille datée au ${ }^{14} \mathrm{C} ; 8$. Extrapolation directe du taux de sédimentation évalué par la datation au ${ }^{210} \mathrm{~Pb} /{ }^{137} \mathrm{Cs}$.

\section{Fig. 2 - Dating results.}

$A:{ }^{210} \mathrm{~Pb}$ profiles according to the depth in cores $B H-B,-C,-D$, et $-E .{ }^{137} C S$ profiles are drawn depending on the age model derived from each core's ${ }^{210} \mathrm{~Pb} b_{x s}$ profile to test its validity. The lines highlight the years 1963 for all cores and also 1986 BH-C core. B: Age models of cores: 1. BH-A and BH-B; 2. BH-C; 3. BH-D; 4. BH-E. 5. Shell dated with ${ }^{14} \mathrm{C}(\mathrm{cm} 62-1810 \mathrm{AD}) ; 6 .{ }^{210} \mathrm{~Pb} /{ }^{137} \mathrm{Cs}$ Dating; 7 . Extrapolation of the sedimentation rate assessed from the end of the ${ }^{210} \mathrm{~Pb} /{ }^{137} \mathrm{Cs}$ dating to the ${ }^{14} \mathrm{C}$ shell dating; 8. Direct extrapolation of sedimentation rate assessed by the ${ }^{210} \mathrm{~Pb} /{ }^{137} \mathrm{Cs}$ dating. 
attendu à environ 1963, en lien avec le maximum des retombées des essais nucléaires atmosphériques, pour les trois carottes. Les activités de ${ }^{137} \mathrm{Cs}$ plus élevées dans la carotte $\mathrm{BH}-\mathrm{C}$ permettent même d'identifier un pic secondaire à 1986, induit par l'accident nucléaire de Tchernobyl.

La coquille marine extraite à $62 \mathrm{~cm}$ dans la carotte $\mathrm{BH}-\mathrm{C}$, et datée par le ${ }^{14} \mathrm{C}$ à $1810 \pm 30$ ans, a été utilisée pour dater les horizons les plus profonds de ces trois carottes. Ceci conduit à un taux de sédimentation moyen de $0,45 \mathrm{~cm} / \mathrm{an}$ entre la base des couches datées par le ${ }^{210} \mathrm{~Pb}_{\mathrm{xs}}$ et le niveau où la coquille a été extraite dans la carotte $\mathrm{BH}-\mathrm{C}$. Ce taux de sédimentation est supérieur à celui du faciès superficiel, dû à la forte proportion de sables présents. Comme les trois carottes sont localisées dans le même environnement et présentent des taux de sédimentation comparables pour les horizons superficiels, nous avons également utilisé ce taux de sédimentation moyen pour extrapoler la datation des horizons les plus profonds des carottes BH-D et BH-E (fig. 2).

$\mathrm{BH}-\mathrm{A}$

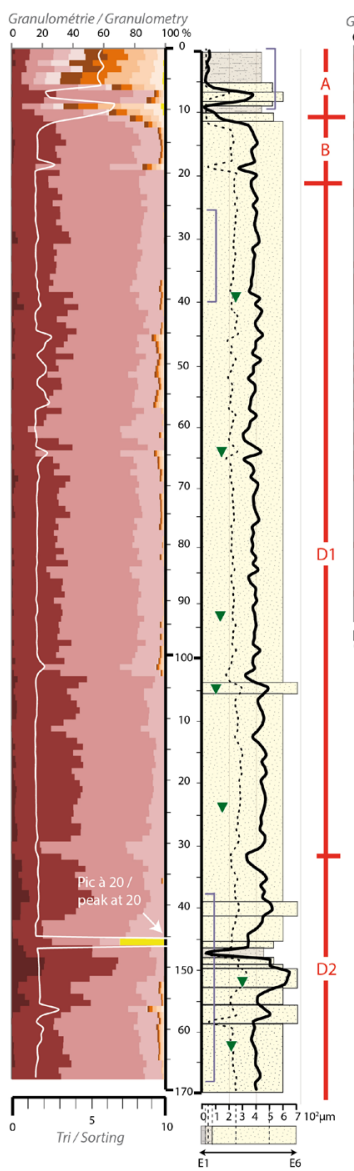

$\mathrm{BH}-\mathrm{B}$

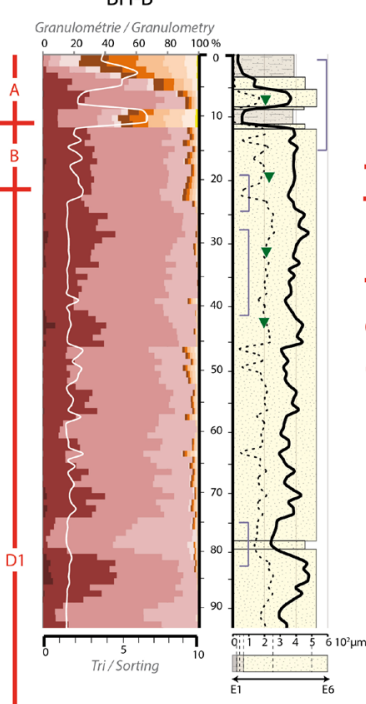

$\mathrm{BH}-\mathrm{C}$

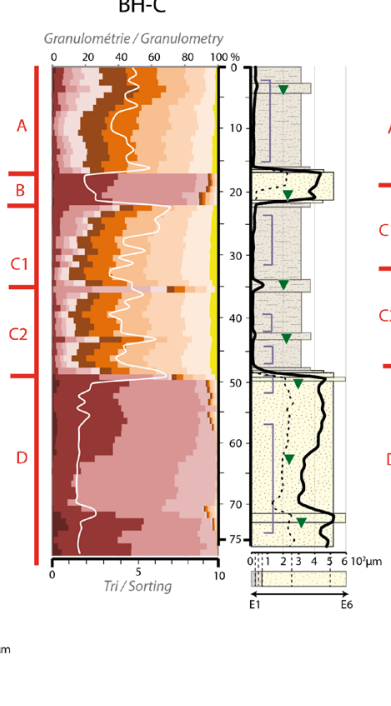

\subsubsection{Datations des carottes $B H-A$ et $B H-B$}

Les deux carottes $\mathrm{BH}-\mathrm{A}$ et $\mathrm{BH}-\mathrm{B}$ prélevées sur la flèche sableuse (que nous appellerons dans cet article flèche $\mathrm{D}$ ) sont principalement composées de sables surmontés par une couche d'environ $10 \mathrm{~cm}$ d'épaisseur d'une argile limoneuse (fig. 1). L'horizon superficiel limoneux de la carotte BH-B a été sélectionné pour dater ces deux carottes, car il est le plus épais. Les activités de ${ }^{210} \mathrm{~Pb}_{\mathrm{xs}}$ sont assez faibles et diminuent rapidement avec la profondeur pour atteindre des niveaux négligeables vers $8-10 \mathrm{~cm}$, indiquant une vitesse de sédimentation inférieure $(0,11 \mathrm{~cm} / \mathrm{an})$ à celles déterminées sur les carottes prélevées au cœur de l'anse des Rouillères. En l'absence de coquilles qui auraient pu permettre de réaliser des datations complémentaires, ce taux de sédimentation moyen a été utilisé pour extrapoler les âges des carottes BH-A et BH-B (fig. 2), car leur log est homogène et comparable (fig. 3). Il est à souligner que la datation des niveaux profonds de ces deux carottes est moins précise que dans le cas des carottes BH-C, BH-D et BH-E.

A, B, C1, C2, D1, D2 : Principaux faciès identifiés / Main facies identified
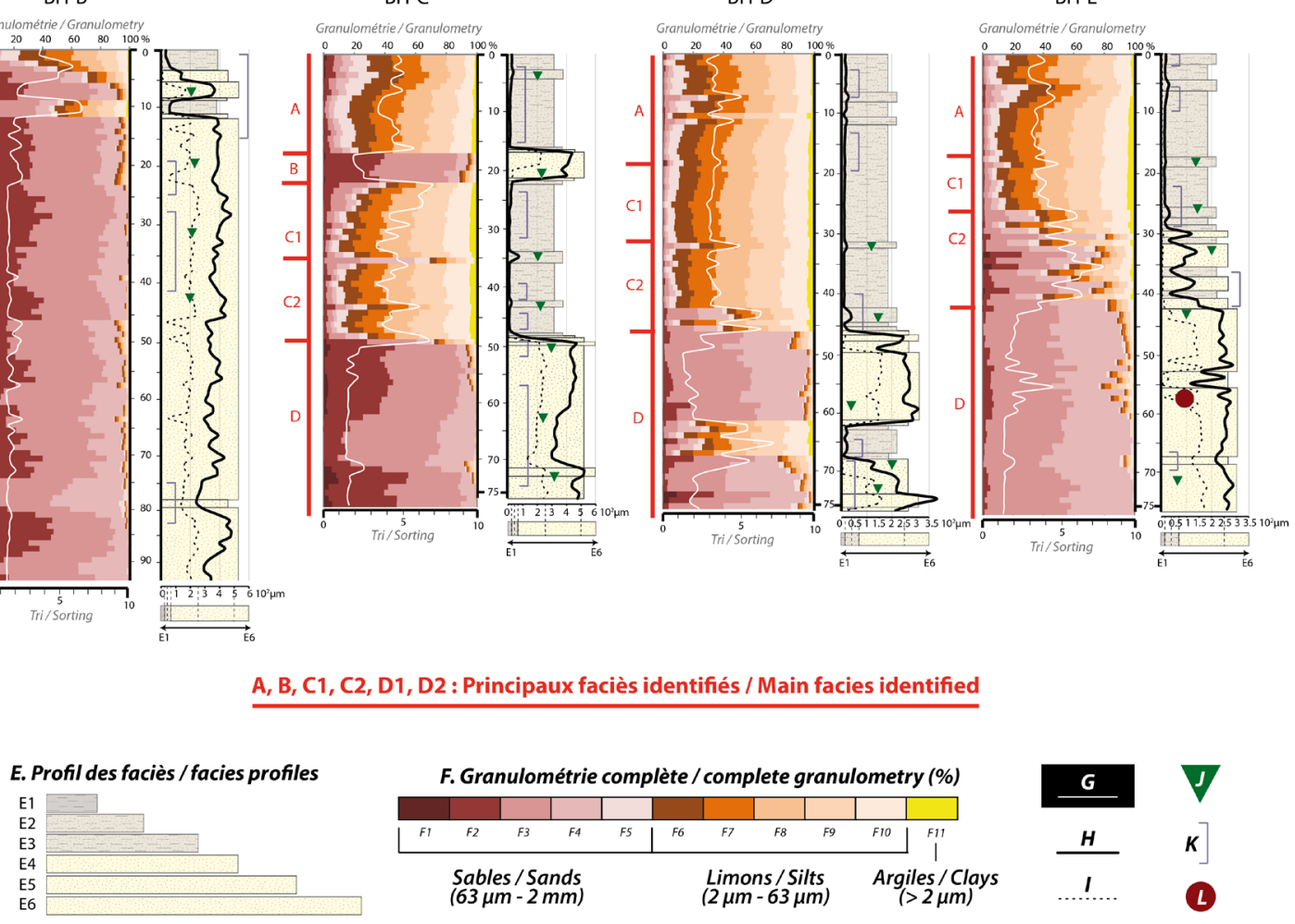

Fig. 3 - Logs des cinq carottes extraites de l'Anse des Rouillères dans la lagune de la Belle-Henriette.

A, B, C1, C2, D1, D2 : Principaux faciès identifiés, dates estimées : A : depuis 1916; B : $1908-1916$; C1 : 1840 - fin du XIX siècle ; C2 : $1820-1840 ;$ D1 (BH-A et BH-B) : $\approx 750$ $\mathrm{AD}$ - fin du XIX siècle; D1 (BH-C, BH-D et BH-E) : ? - début du XIX siècle; D2 : antérieur à $\approx 750$ AD ; E. Profil des Faciès (sédiment dominant) : E1. Argiles limoneuses; E2. Limons argileux ; E3. Limons sableux ; E4. Sables fins ; E5. Sables moyens ; E6. Sables grossiers ; F. Granulométrie complète (\%) : F1. Sables très grossiers ; F2. Sables grossiers ; F3. Sables moyens ; F4. Sables fins ; F5. Sables très fins ; F6. Limons très grossiers ; F7. Limons grossiers ; F8. Limons moyens ; F9. Limons fins ; F10. Limons très fins ; F11. Argiles ; G : Indice de tri $(0-10) ; \mathrm{H}$ : Grain moyen $(\mu \mathrm{m}) ; \mathrm{I}$ : Dixième décile $(\mu \mathrm{m}) ; \mathrm{J}$ : Coquilles extraites ou débris coquilliers; $\mathrm{K}$ : Forte matière organique; $\mathrm{L}$ : Elément grossier marquant extrait.

Fig. 3 - Logs of the five cores extracted from Anse des Rouillères in the Belle-Henriette lagoon.

$A, B, C 1, C 2, D 1, D 2:$ Main facies identified, estimated dates: A: since 1916; B: $1908-1916 ; C 1: 1840$ - end of the 19 th century; C2: $1820-1840 ; D 1$ (BH-A et BH-B): $\approx 750$ AD - end of the $19^{\text {th }}$ century; D1 (BH-C, BH-D et BH-E): ? - early $19^{\text {th }}$ century; D2: previous to $\approx 750$ AD; E. Facies profiles (main sediment): E1. Silty clays; E2. Clayey silts; E3. Sandy silts; E4. Fine sands; E5. Medium sands; E6. Coarse sands; F. Complete granulometry (\%): F1. Very coarse sands; F2. Coarse sands; F3. Medium sands; F4. Fine sands; F5. Very fine sands; F6. Very coarse silts; F7. Coarse silts; F8. Medium silts; F9. Fine silts; F10. Very fine silts; F11. Clays; G: Sorting index (0-10); H: Mean grain size ( $\mu$ m); I: Tenth decile ( $\mu$ m); J: Shells extracted or shell remains; K: High organic matter; L: Large pebble extracted. 


\subsection{Caractéristiques sédimentologiques des carottes prélevées}

\subsubsection{Carottes $B H-C, B H-D$ et $B H-E$}

Les trois carottes BH-C, BH-D et BH-E prélevées en 2019 au cœur de l'anse des Rouillères sur une slikke (fig. 1) ont une structure lithostratigraphique similaire (fig. 3). Un premier horizon A commun à l'ensemble des trois logs est identifié sur la structure superficielle comprenant les 16 premiers centimètres des trois carottes. Ce niveau limoneux et riche en matière organique, correspondant à la surface actuelle du substrat de l'anse des Rouillères, présente un grain moyen d'environ $40 \mu \mathrm{m}$ hétérogène (indice de tri de 5 à 6). Ce premier ensemble est interrompu par plusieurs niveaux de silts grossiers aux centimètres 4 (BH-C, daté à environ 1999), 6-7 (BH-D daté à environ 1999 et BH-E à environ 1970) et 11 (BH-D daté à environ 1940). Plusieurs événements extrêmes sont survenus à ces dates dans l'ouest de la France (Pouzet et al., 2019). Un second horizon B est seulement visible dans le log de la carotte BH-C, composé de sables moyens homogènes d'environ $400 \mu \mathrm{m}$ (taux de sables atteignant $90 \%$ ) entre les centimètres 16 (daté à 1916) et 22 (daté à 1908).

Les trois logs stratigraphiques présentent ensuite des paramètres comparables entre les centimètres 22 (daté à environ 1896) et 44 pour BH-E, 46 pour BH-D, et 48 pour BH-C (datés entre 1820 et 1840). Bien que la composition globale du faciès $C$ puisse sembler similaire au faciès $\mathrm{A}$, nous relevons une présence bien plus importante d'argiles atteignant les $10 \%$ dans l'horizon $\mathrm{C}$, tandis qu'elle reste assez constante et inférieure à $5 \%$ dans le niveau superficiel A. Un premier sous-ensemble $\mathrm{C} 1$ témoigne de conditions morphogéniques calmes, avec un sédiment limoneux pauvre en sables, transitant ensuite vers un environnement plus dynamique $\mathrm{C} 2$, alternant entre limons fins, limons grossiers et sables fins. Nous notons ici une double progression, tout d'abord dans la dynamique de la couche $\mathrm{C} 2$, avec une hétérogénéité bien plus marquée dans le log de la carotte BH-E. Par ailleurs, nous relevons également une évolution entre les trois carottes sur l'épaisseur de ce niveau C2, plus fin dans le log de la carotte BH-E. En s'écartant du cœur de la lagune, il y a une plus grande hétérogénéité et une diminution de l'épaisseur de ce niveau, transitant ensuite plus rapidement vers le faciès sableux D, estimé autour de 1820 .

Enfin, le dernier faciès D témoigne d'un horizon profond sableux entre les centimètres 44 et 48 (datés à 1820-1840) et la base des carottes au centimètre 76 daté à 1780-1800. Les sables dominent très largement avec plus de $90 \%$ de la composition du sédiment généralement bien trié, excepté pour les deux occurrences ponctuelles de sédiments plus fins (silts ou sables fins) observées aux centimètres 62-68 de la carotte BH-D et 54-56 de la carotte BH-E. Une distinction peut être faite dans la composition sableuse entre ces trois logs, avec une proportion de sables grossiers et très grossiers plus importante au sein de la carotte $\mathrm{BH}-\mathrm{C}$, pouvant atteindre $50 \%$ de la composition sédimentaire totale.

\subsubsection{Carottes $B H-A$ et $B H-B$}

Les deux carottes BH-A et BH-B prélevées en 2018 sur la flèche sableuse (fig. 1) présentent des logs comparables (fig. 3). L'horizon A superficiel est composé d'une couche dominée par des limons grossiers (avec un grain moyen allant de 40 à $60 \mu \mathrm{m}$ ) jusqu'aux centimètres 10-12 (datés à 1900-1920). Ce faciès est semblable au niveau A de BH-C, BH-D et BH-E, mais est peu épais. Ce premier horizon est interrompu par un niveau de sables moyens $(\approx 350 \mu \mathrm{m})$ entre les centimètres 5 et 7 (daté vers 1940). Cette occurrence sableuse peut être expliquée par un événement extrême ponctuel déjà observé dans la région (Pouzet et al., 2019). En excluant cette occurrence marine ponctuelle, le premier faciès $\mathrm{A}$, dominé par les limons, est composé de sédiments hétérogènes (indice de tri d'environ 5) et riches en matière organique, car le site de prélèvement humide et composé de silts accueille une végétation de schorre submergée aux marées de vives eaux.

À partir des centimètres 10-12 et jusqu'aux niveaux les plus profonds, les sables fins à grossiers dominent largement dans un ensemble sédimentologique beaucoup plus homogène (indice de tri autour de 1). La composition sableuse atteint des proportions supérieures à $90 \%$ sur la majeure partie de cet horizon. Au sein de cet ensemble, une sous-distinction peut être faite entre un premier faciès $\mathrm{B}$, composé de sables passant progressivement d'un grain fin à moyen jusqu'aux centimètres 20-25 (daté 1800-1820), avec quelques variations nettes dans l'indice de tri et de grain moyen (cm. 18 de BH-A, 14 à 22 de BH-B). Ce faciès présente une structure comparable au sédiment du faciès $\mathrm{B}$ détecté de la carotte BH-C. Nous relevons ensuite un second faciès sableux D1 composé de sables moyens atteignant $400 \mu \mathrm{m}$ jusqu'au centimètre 135 (évalué avec de fortes incertitudes aux horizons $750 \mathrm{AD}$ ), et un troisième niveau D2 avec des éléments plus grossiers (atteignant jusqu'à $600 \mu \mathrm{m})$, mais également plus riches en matière organique, et marqués par une très brève rupture avec un horizon fin de limons (centimètre 147) jusqu'au fond de la carotte BH-A. Les proportions de sables "très grossiers " et "grossiers " atteignent respectivement des valeurs plus importantes dans le faciès D2 entre 135 et $170 \mathrm{~cm}$ (10 et 50\% respectivement) que dans les horizons D1 entre 25 et $135 \mathrm{~cm}$ (moins de $5 \%$ et $30 \%$ respectivement) et B entre 12 et $25 \mathrm{~cm}$ (grains très grossiers quasi nuls et grossiers autour de $30 \%$ ). Les caractéristiques sédimentologiques de l'horizon D1 sont comparables à celles du niveau $\mathrm{D}$ des trois carottes $\mathrm{BH}-\mathrm{C}, \mathrm{BH}-\mathrm{D}$ et BH-D. Elles se rapprochent particulièrement du faciès $\mathrm{D}$ de la carotte $\mathrm{BH}-\mathrm{C}$, composée de sables plus grossiers que les faciès $\mathrm{D}$ des carottes $\mathrm{BH}-\mathrm{D}$ et $\mathrm{BH}-\mathrm{E}$.

\subsection{Vestiges archéologiques}

Le secteur de la lagune de la Belle-Henriette offre peu de vestiges archéologiques, hormis des murs de pierre sèche établis par des particuliers sur l'estran, représentant des restes d'anciennes pêcheries littorales ou écluses à poisson. Les estrans de l'ouest de la France possèdent des vestiges de pièges à poissons, nommés " pêcheries " réalisés en bois ou, comme c'est le cas majoritairement dans la région, en pierre (Daire et Langouët, 2010). Il s'agit d'amas plus ou moins linéaires disposés méthodiquement, en utilisant les roches émergentes, de façon à réaliser un barrage qui va piéger le poisson et l'obliger à se diriger vers une porte qui dispose souvent d'un moyen de collecte du poisson. Leur ancienneté est toujours discutée, mais leur positionnement sur l'estran a permis d'établir un indice de datation : elle est dite d'une grande ancienneté lorsqu'une pêcherie est entièrement noyée même à marée basse de fort coefficient. Plusieurs recherches réalisées en Bretagne, au Royaume- 
Uni et au Danemark montrent que ces dernières peuvent remonter au Néolithique, à l'Âge du bronze, voire au Mésolithique. Leur étude permet d'avoir un éclairage sur les modalités d'exploitation des ressources littorales et sur les interactions Homme-milieu des sociétés anciennes (Daire et Langouët, 2011).

Cette pratique commune des sociétés littorales est attestée depuis la Préhistoire récente et se poursuit durant l'époque contemporaine. Avec l'Ordonnance de Colbert en 1681 et la création du Domaine Publique Maritime, ces structures, alors apanages de sociétés littorales agricoles, sont placées sous la juridiction de l'État. Dès lors, leur usage décroît progressivement dans un souci de gestion raisonnée des ressources (Masson Du Parc réed. 2009). La présence de pêcheries sur l'estran est documentée par l'archéologie en Normandie (Billard et Bernard 2016), Bretagne (Daire et Langouët 2008, 2010), Pays de la Loire (Soulet 1995 ; Tessier 2004 ; Large et al. 2009 ; Langouët et al., 2010 ; Petuya 2019 ; Gruet et Borvon 2022) ou encore en Nouvelle-Aquitaine, notamment à l'île de Ré (Boucard 1984). Récemment, les pêcheries vendéennes de l'île de Noirmoutier et de l'île d'Yeu ont fait l'objet d'une synthèse et d'une actualisation dans le cadre d'un mémoire de master soutenu à l'Université de Nantes (Petuya, 2019).

Sur la commune de la Tranche-sur-Mer, trois pêcheries en pierre sont encore répertoriées en 1855, période d'inventaire pour le renforcement de la législation relative au Domaine Public Maritime. Une première pêcherie est située à la Pointe du Bureau, structurée en arc de cercle irrégulier sur une étendue de 200 ares. La seconde est en pentagone à la Pointe du Cimetière, convexe sur une étendue de 230 ares. Une troisième pêcherie se trouve à la Pointe de la Grière, et s'étend sur une surface de 180 ares de forme rectangulaire. Ces trois pêcheries étaient ainsi encore en usage au milieu du XIX ${ }^{e}$ siècle. Par ailleurs, les travaux de prospection littorale réalisés par Large en 2010 ont mis en exergue la présence de dix empierrements en roche allochtones de forme arciforme et quadrangulaire non recensés (fig. 4A). Ces structures s'appuient sur les platiers rocheux parfois en connexion avec la plage actuelle. L'installation de ces pêcheries n'a pas été datée. La seule certitude est un emploi massif sur le long terme jusqu'à l'établissement du Domaine Publique Maritime, avec un arrêt total de leur exploitation dans les années 1990. À partir de cette cartographie, la pêcherie qui est recensée en 1855 à la Pointe de la Grière semble être celle qui a été identifiée la plus à l'est par Large en 2010. Ainsi, cette pointe est également appelée aujourd'hui Pointe $d u$ Rocher le long de la plage de la Grière, un lien toponymique peut donc être établi (fig. 4A-B). Elle est donc située à approximativement deux kilomètres à l'ouest de l'anse des Rouillères. Concernant les deux autres qui ont été référencées en 1855 , aucun lien toponymique ne peut être établi avec les travaux de Large en 2010. Alors que les neuf autres empierrements ont été localisés à proximité de l'ensemble rocheux du Groin au Cou, nous émettons l'hypothèse, sans certitude, que ces deux pêcheries pourraient correspondre à deux de ces neuf empierrements situés les plus à l'ouest de cette cartographie (fig. 4A).
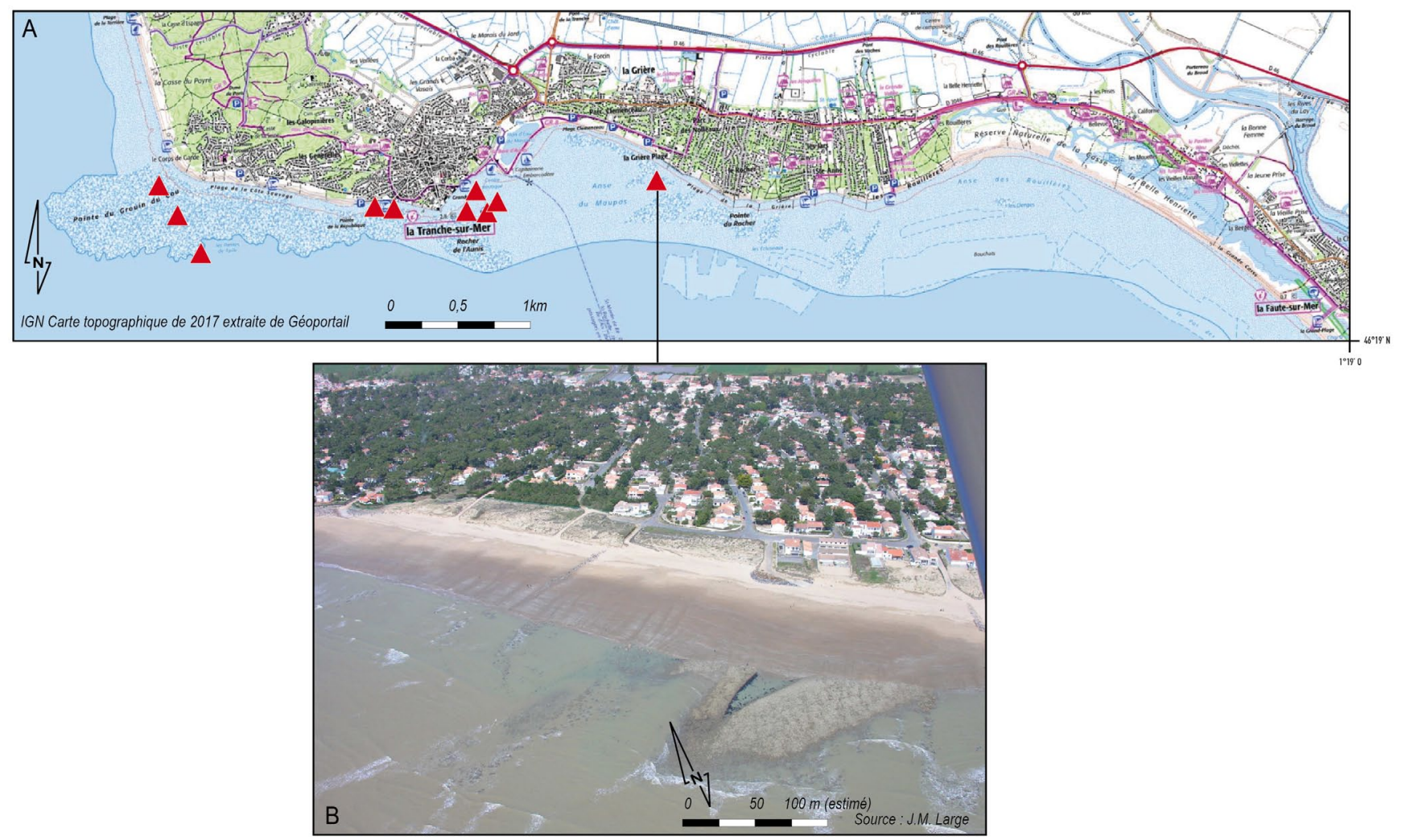

Fig. 4 - Vestiges archéologiques.

A : Position des dix empierrements recensés (triangles rouges) à La-Tranche-sur-Mer par Large en 2010 ; B : photographie prise par ULM de la pêcherie située à proximité de la Pointe de la Grière.

Fig. 4 - Archaeological remains.

A: Position of the ten enstonements inventoried (red triangles) in La-Tranche-sur-Mer by J-M. Large in 2010; B: Photograph of the fisheries located next to Pointe de la Grière taken from ultralight aviation. 


\section{Discussion}

\subsection{Reconstruction paléoenvironnementale de la lagune de la Belle-Henriette avec l'apport des cartes anciennes}

Par le croisement des données sédimentologiques issues des cinq carottes prélevées et de l'étude des cinquante-cinq cartographies historiques consultées, nous présentons une évolution paléoenvironnementale allant de la construction de la lagune de la Belle-Henriette jusqu'aux prémices du Marais poitevin, durant la période antérieure à son comblement.

\subsubsection{Lancien Golfe des Pictons et la migration du Lay}

Le premier environnement le plus ancien présenté par cette étude est seulement extrait des cartographies historiques, car il ne pourrait être identifié qu'en réalisant des carottages allant jusqu'à des profondeurs d'environ vingt mètres. Le secteur sur lequel est aujourd'hui implantée la lagune de la Belle-Henriette est un ancien golfe maritime appeléle « Golfe des Pictons », ancienne plaine devenue submergée durant la transgression marine liée à l'interglaciaire Holocène. Plusieurs paroisses, devenues des communes telles que Champagné-les-Marais, Grues, Saint-Michel-en-l'Herm ainsi que le lieu-dit de la Dive, étaient alors insulaires, tandis que les communes littorales de La-Faute-sur-Mer et de l'Aiguillon-sur-Mer, implantées suite au colmatage du marais, étaient alors inexistantes (fig. 5A).
À partir de la base de données en ligne Infoterre (http://infoterre. brgm.fr) du Bureau de Recherches Géologiques et Minières (BRGM), une campagne de cinq carottages profonds (allant jusqu'à environ 22 mètres) de 1984 est analysée. Comme le montre l'une d'entre elles prélevée dans l'actuelle lagune de la BelleHenriette identifiée BSS001PEUL (http://ficheinfoterre.brgm.fr/ InfoterreFiche/ficheBss.action?id=BSS001PEUL), nous notons la présence d'un faciès présent entre 19 et 20 mètres correspondant aux alluvions du Lay, anciennement appelé Saint-Benoist. Au sein des sondages, ces niveaux varient entre 14 et $20 \mathrm{~m}$ de profondeur. Ils témoignent des migrations de l'estuaire du Lay, impulsées par la progradation de la flèche sableuse $\mathrm{C}$ qui a isolé le marais de l'océan au niveau de son ancien estuaire. Son tracé semble donc avoir migré vers le sud-est durant le comblement de l'ancien golfe, jusqu'à sa position actuelle entre les deux flèches B et C. Cependant, aucune datation radio-isotopique ne permet aujourd'hui d'évaluer les périodes durant lesquelles ces dynamiques se sont produites. Le Lay a largement contribué au colmatage de cette ancienne baie, tapissant le sud-ouest du Golfe des Pictons d'alluvions (fig. 5B). Durant ces périodes anciennes estimées à l'Antiquité et au Moyen Âge, les flèches sableuses A (flèche la plus large et haute allant des actuelles communes de Jard-sur-Mer à la Tranche-sur-Mer) et B (actuelle pointe de l'Aiguillon) se sont formées pour dessiner le littoral et fermer cet ancien marais de l'océan durant le colmatage (fig. 5B). Les travaux de Large (Large et al., 2017) nous apprennent que la mise en place de la flèche A dans sa position actuelle est postérieure à 540-
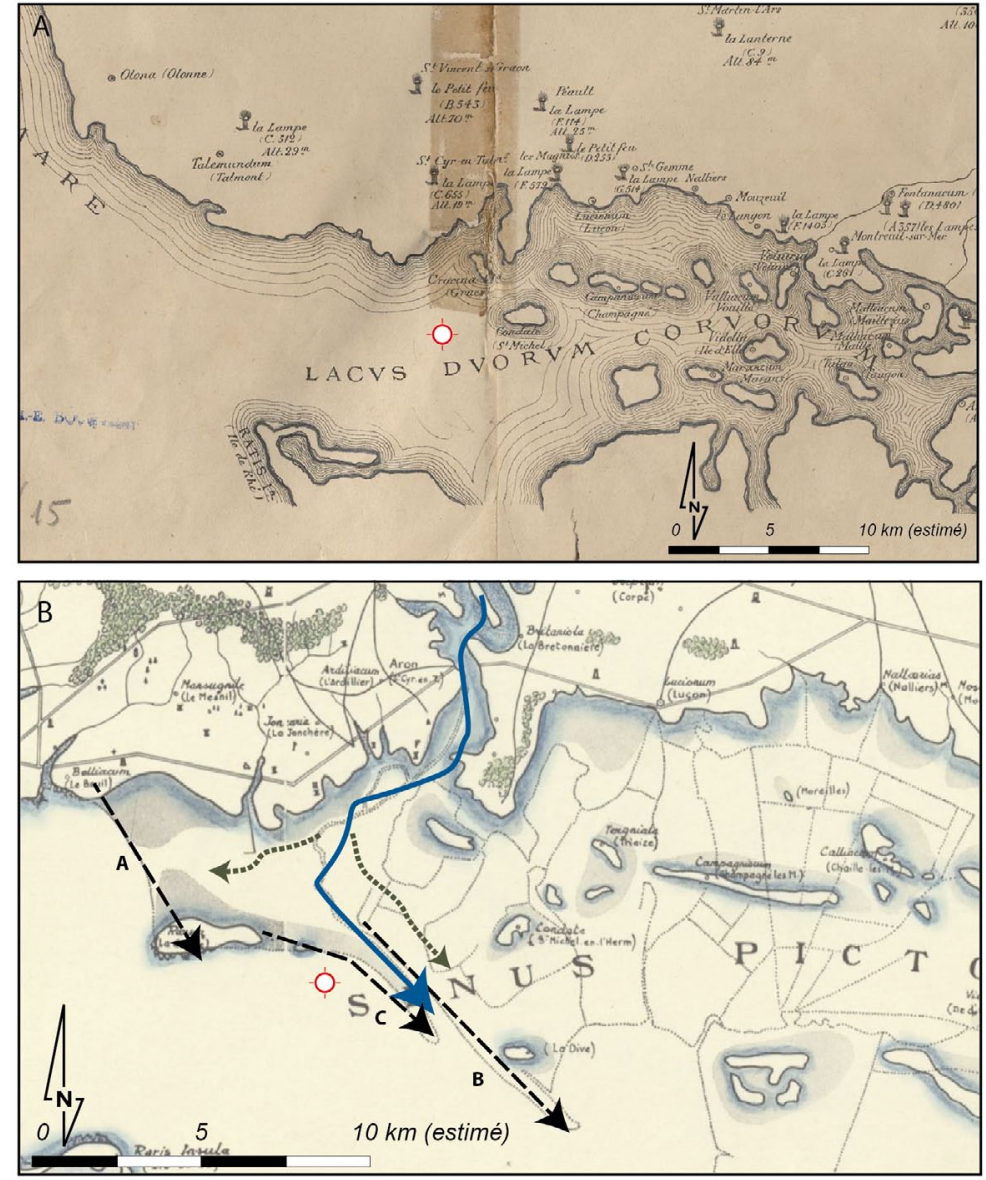

C

- 1

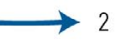

Fig. 5 - Évolution diachronique du Golfe des Pictons entre l'Antiquité et le Moyen Âge.

A : Extrait de la cartographie intitulée "Les phares du littoral BasPoitevin dans l'Antiquité", Archives Départementales de Vendée, référence $7 \mathrm{Fi} 1506$ (Contexte historique estimé : “Antiquité") ; B : Extrait de la cartographie intitulée "Carte archéologique du BasPoitou", Archives Départementales de Vendée, référence 7 Fi 527 (Contexte historique estimé : "Préhistoire / Antiquité / Moyen Âge»); $\mathrm{C}$ : Légende : 1 . Sites de carottage (approximatifs) ; 2. Futur tracé du Lay (ex- Saint Benoist) ; 3. Futur comblement alluvionnaire ; 4. Futures progradations des flèches sableuses $\mathrm{A}, \mathrm{B}$ et $\mathrm{C}$.

Fig 5 - Diachronic evolution of the Gulf of Pictons between the Antiquity and the Middle Ages.

A: Excerpt from the cartography entitled "Les phares du littoral Bas-Poitevin dans l'Antiquité", Departmental Archives of Vendée, reference 7 Fi 1506 (Estimated historical context : "Antiquité"); B: Excerpt from the cartography entitled "Carte archéologique du Bas-Poitou", Departmental Archives of Vendée, reference 7 Fi 527 (Estimated historical context: "Préhistoire / Antiquité / Moyen Âge»; C: Legend: 1. Coring sites (estimation); 2. Future Lay (former Saint Benoist) stream; 3. Future alluvial filling; 4 . Future progradation of the sandy spits $A, B$ and $C$. 
230 BC ( $\approx 2490-2180$ BP), soit au Second Âge du Fer. Cette hypothèse résulte d'une datation au ${ }^{14} \mathrm{C}$ obtenue sur une brindille enchâssée dans du bri dans la partie est de la plage du Rocher à Longeville-surMer, située dans la partie la plus septentrionale de la flèche A. Pour la partie méridionale et orientale du marais poitevin non discutée dans cette étude, l'apport d'alluvions par Sèvre Niortaise a aussi contribué au colmatage de cette ancienne baie maritime.

\subsubsection{L'implantation d'une baie maritime par la construction de la flèche sableuse $C$}

Les premières représentations cartographiques de cet environnement sont approximatives, mais elles permettent d'évaluer la position de l'ancien estuaire du Lay (ex St-Benoist) par rapport à la flèche $\mathrm{B}$. Bien que comportant quelques incohérences, la représentation de 1627 issue de Chapuis, 2007 l'illustre nettement, avec un estuaire estimé à quelques centaines de mètres à l'ouest du site de carottage (fig. 6A). L'environnement étudié au niveau du secteur carotté est donc passé d'une ancienne baie maritime à une séquence de progradation progressive de la flèche sableuse $\mathrm{C}$, favorisant la sédimentation des marais situés en arrière. Les horizons les plus profonds des trois carottes lagunaires sont principalement composés de sables marins, et témoignent donc de la présence de l'ancienne baie et de la construction progressive de la flèche $\mathrm{C}$ que nous pouvons identifier sur chacune des cartes historiques (fig. 6). Selon les datations, les carottes BH-C, BH-D et BH-E nous offrent seulement un recul temporel sur les trois derniers siècles, le faciès $\mathrm{D}$ identifié correspond donc à la progradation de cette flèche (fig. 3). Cette progradation semble s'effectuer par phases, avec notamment des variations de granulométrie principalement identifiées dans les carottes BH-D et BH-E. Les deux carottes BH-A et BH-B nous permettent de remonter plus loin dans le temps avec notamment la distinction entre deux niveaux sableux distincts (fig. 3) évaluée à $750 \mathrm{AD}(1200 \mathrm{BP})$. Nous ne pouvons pas émettre d'hypothèses sur l'initiation de la progradation de cette flèche, mais il semble que l'espace était bien occupé par l'ancienne baie maritime, et qu'il a migré vers la formation de la flèche $\mathrm{C}$ durant l'ensemble de la phase représentée par le faciès $\mathrm{D}$. La légère modification des conditions morphogéniques observée au $\mathrm{cm}$. 135 et estimé à $750 \mathrm{AD}$ peut être due à une bascule dans le fonctionnement de l'ancienne baie maritime, ayant conduit à la formation de la flèche durant les siècles suivants. Les différentes phases de progradation de la flèche sableuse C sont finement présentées dans les travaux de J. Allard et C. Poirier (Allard, et al., 2008; Poirier et al., 2017).

\subsubsection{L'environnement temporaire de l'Anse des Terres Noires}

Entre la fin du XVIII ${ }^{e}$ siècle et les années 1900, le paysage était occupé par l'" Anse des Terres Noires ». La flèche C montrait de fortes variations morphologiques durant sa progradation comme l'illustrent les cartographies anciennes de Cassini de 1740 (fig. 6B), et la cartographie de 1757 (fig. 6C). En conséquence, un bras de mer temporaire a notamment été identifié sur les anciennes cartes, menant jusqu'à l'estuaire de la rivière du Lay, où se trouvait le lieudit du «Port Puant». Cette configuration explique l'environnement vaso-limoneux riche en matière organique observé dans les trois carottes situées au cœur de la lagune (faciès $\mathrm{C} 1$ ), et les arrivées alternatives de limons et de sables dans la carotte BHE située près de la flèche $C$ (faciès C2), attestant d'arrivés tantôt sableuses et tantôt limoneuses des sédiments durant la progradation de la flèche C (fig. 3).

La carte ancienne de 1824 soutient cette hypothèse (fig. 6G-1). La situation de la brèche conduit à une alternance d'apports, avec une sédimentation dominée par des limons provenant de la rivière dans l'espace de la future lagune, entrecoupée par des incursions marines guidées par la progradation de la flèche C. Ces apports ont été détectés par les alternances très significatives de grain, entre niveaux sableux marins et vaso-limoneux fluviaux dans les faciès C2. Ces fortes variations granulométriques sont notamment identifiées dans la carotte $\mathrm{BH}-\mathrm{E}$ située au plus près de la flèche $\mathrm{C}$. À l'inverse, le faciès $\mathrm{C} 2$ des carottes $\mathrm{BH}-\mathrm{C}$ et $\mathrm{BH}-\mathrm{D}$ situées plus à distance de la flèche $\mathrm{C}$ semble plus stable avec des niveaux majoritairement limoneux (fig. 3). Les toponymes "Anse des Terres Noires " et " Port Puant » inscrits dans ces cartes (fig. 6G-2 et G-3), témoignent de cet environnement «boueux » détecté dans les trois carottes, au sein duquel une zone marécageuse temporaire était installée. Le terme « molin » dans le "langage du pays » est également retrouvé pour caractériser ces ensembles vaseux de couleur noire ou grise (Bouhier, 1957). Enfin, les travaux de Fustec-Mathon et Mathon discutaient également de ces hypothèses dans les années 1960: "L'installation de bouchots favorise l'ensablement de cette flèche [Pointe ou flèche d'Arcay, ici nommée flèche $\mathrm{C}]$ constituée d'une série de crochets sableux émergés successivement et qui se sont joints, laissant entre eux des dépressions vaseuses et vaso-sableuses. Ces laisses vaseuses ainsi que la partie de la Flèche d'Arçay bordant le Lay, la lagune située derrière le cordon littoral de la Belle-Henriette et l'ancienne embouchure du Lay au lieu-dit Port Puant, abritent une végétation halophile de terrains vaseux et vaso-sableux qui ne font pas l'objet de la présente note." (Fustec-Mathon et Mathon, 1960).

L'implantation de l'Anse des Terres Noires a été datée à approximativement 1820 selon le taux de sédimentation évalué entre la datation ${ }^{210} \mathrm{~Pb} /{ }^{137} \mathrm{Cs}$ et la coquille datée au ${ }^{14} \mathrm{C}$ au niveau du faciès C2 (fig. 3), ce qui concorde avec les documents cartographiques historiques analysés datant principalement de la première moitié du XIX siècle et de la fin du XVIII ${ }^{e}$ siècle. Ces mentions sont notamment présentes sur le cadastre napoléonien (fig. 6G-2) et la carte de Claude Masse (fig. 6G-3), alors que l'ouverture entre l'océan et le Port Puant est bien visible sur les cartographies historiques de la fin du XVIII (fig. 6D), du cadastre napoléonien (fig. 6E), des cartographies de 1818 (fig. 6F) et de 1824 (fig. 6G-1).

\subsubsection{La configuration lagunaire actuelle après la construction de la flèche sableuse D}

La dernière grande évolution morphologique caractérisant cet espace est la construction de la flèche sableuse D qui a isolé la lagune de l'océan. D'après les datations du faciès $\mathrm{B}$ de la carotte BH-C située au centre de la lagune, cette flèche sableuse D aurait été formée au début du $\mathrm{XX}^{\mathrm{e}}$ siècle au niveau de l'Anse des Rouillères (fig. 2-3). Cette hypothèse est confirmée par l'analyse des données historiques, car cette flèche est absente des cartes produites avant 1900 (fig. 7A-B), alors que la reconstruction issue de la première campagne de photographie aérienne prise dans ce secteur lors de la mission de 1924 issue des fonds de Verger 

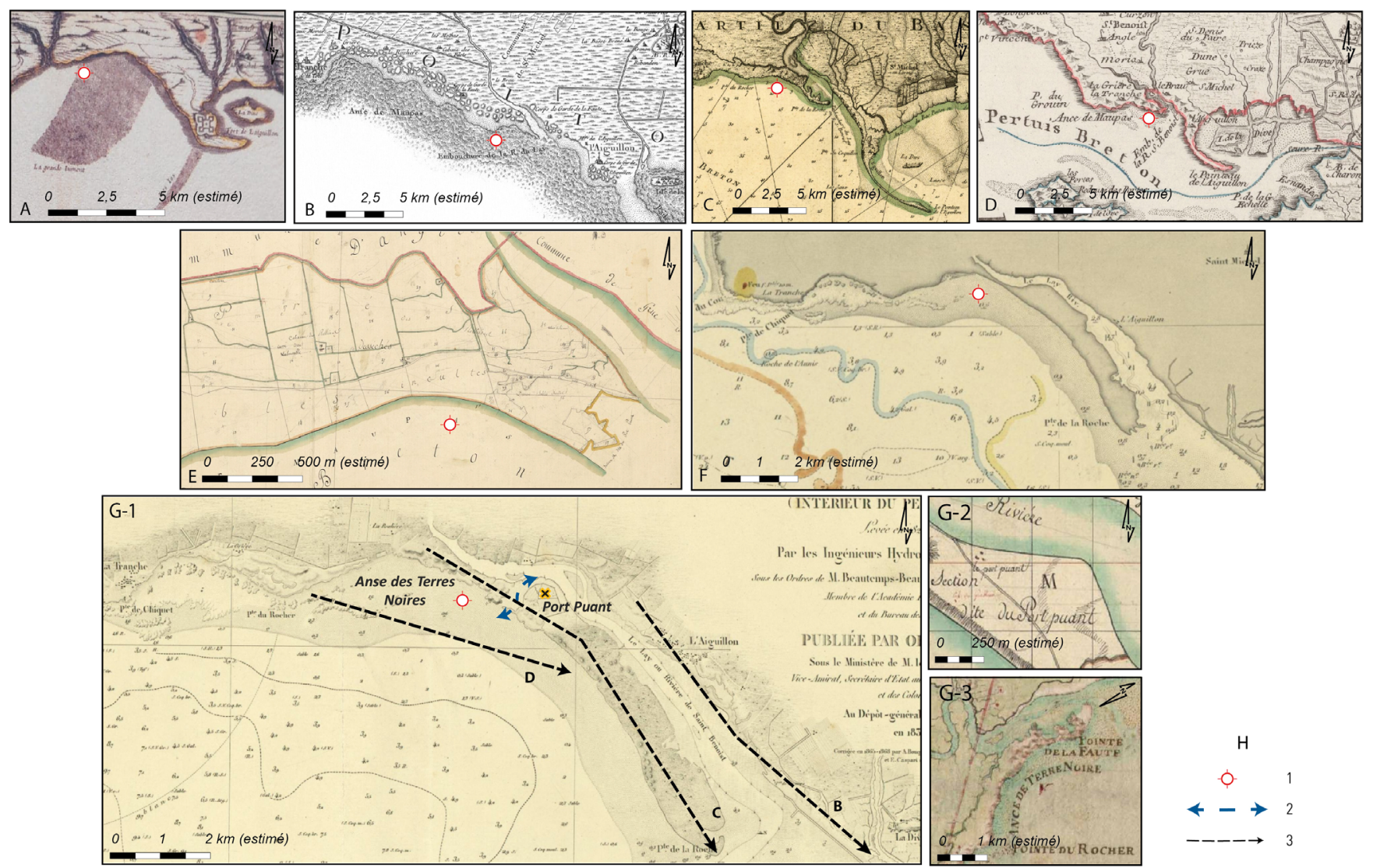

Fig. 6 - Évolution diachronique de l'ancienne « Anse des Terres Noires».

A : Extrait de la cartographie "Carte Anonyme des côtes de Poitou, Aunis et la Rochelle, de l'île de Ré et ses forts (1627)" tirée de Chapuis, 2007 ; B : Carte de Cassini "FEUILLEDERE(ILEDE)N¹33" réalisée en 1740, issue du site internet IGN Remonter le Temps (https://remonterletemps.ign.fr/) ; C : Extrait de la cartographie " Carte des isles [sic] de Ré et d'Olléron, les pertuis d'Antioche, Breton et de Maumusson avec partie des costes [sic] de Poitou, Aunis et Saintonge / dressée au Dépost des cartes et plans de la Marine pour le service des vaisseaux du Roy, par ordre de M. de Moras, ministre et secrétaire d'État ayant le Département de la Marine; par le Sieur [Jacques-Nicolas] Bellin, ingénieur de la Marine et du Dépost des plans... [1757] " Archives Départementales de Vendée, référence 24 Fi 99; D : Extrait de la cartographie "Carte nouvelle de Poitou et du pays d’Aunis..." éditée en 1787 par "chés Crépy», Gallica - Bibliothèque nationale de France, identifiant ark:/12148/btv1b53099565q ; E : Extrait du cadastre napoléonien de La-Tranche-sur-Mer réalisé en 1811, «Section L de la Cabane des Rollières", Archives Départementales de Vendée, référence 3 P 294-9; F : Extrait de la cartographie " Carte des côtes de France, partie comprise entre L'Île-d'Yeu et Chassiron, $n^{\circ} 150$ / levée en 1818-1819», Archives Départementales de Vendée, référence 7 Fi 527 ; G-1 : Extrait de la cartographie « Carte particulière des côtes de France (intérieur du pertuis Breton), $n^{\circ} 156$ / levée en 1822 et 1824, par les ingénieurs hydrographes de la Marine, sous les ordres de M. [Charles-François] BeautempsBeaupré ", Archives Départementales de Vendée, référence 24 Fi 76 (Dépôt de la ville de Luçon); G-2 : Mention du "Port Puant » visible sur le cadastre napoléonien de La-Tranchesur-Mer réalisé en 1811, «Tableau d'assemblage 1811 ", Archives Départementales de Vendée, référence 3 P 294-1 ; G-3 : Mention de l' "Anse des terres Noires " visible sur un extrait de la carte de Claude Masse "Partie du Bas Poitou, générale » réalisée au début du XVIIIe siècle extrait de Suire, 2017 ; H : Légende : 1 . Sites de carottage ; 2 . Brèche / Ouverture vers l'océan ; 3. Progradations des flèches sableuses B et C, et future progradation de la flèche sableuse D.

Fig. 6 - Diachronic evolution of the former "Anse des Terres Noires".

A: Excerpt from the cartography "Carte Anonyme des côtes de Poitou, Aunis et la Rochelle, de l'T̂le de Ré et ses forts (1627)" extracted from the book of Chapuis, 2007; B: Cassini map "FEUILLEDERE(ILEDE)N¹33" created in 1740, extracted from the website IGN Remonter le Temps (https://remonterletemps.ign.fr/); C: Excerpt from the cartography "Carte des isles [sic] de Ré et d'Olléron, les pertuis d'Antioche, Breton et de Maumusson avec partie des costes [sic] de Poitou, Aunis et Saintonge / carried out in Dépost des cartes et plans de la Marine pour le service des vaisseaux du Roy, par ordre de M. de Moras, ministre et secrétaire d'État ayant le Département de la Marine; par le Sieur [Jacques-Nicolas] Bellin, ingénieur de la Marine et du Dépost des plans... [1757]" Departmental Archives of Vendée, reference 24 Fi 99; D: Excerpt from the cartography "Carte nouvelle de Poitou et du pays d'Aunis..." edited in 1787 by "chés Crépy", Gallica - Bibliothèque nationale de France, login detail ark:/12148/btv1b53099565q; E: Excerpt from the Napoleonian cadastral plan of La-Tranchesur-Mer, which was made in 1811, "Section L de la Cabane des Rollières", Departmental Archives of Vendée, reference 3 P 294-9; F: Excerpt from the cartography "Carte des côtes de France, partie comprise entre L'Ile d'Yeu et Chassiron, ${ }^{\circ} 150$ / levée en 1818-1819”, Departmental Archives of Vendée, reference 7 Fi 527; G-1: Excerpt from the cartography “Carte particulière des côtes de France (intérieur du Pertuis Breton), $n^{\circ} 156$ / levée en 1822 et 1824, par les ingénieurs hydrographes de la Marine, sous les ordres de M. [Charles-François] Beautemps-Beaupré", Departmental Archives of Vendée, reference 24 Fi 76 (Luçon municipality depot); G-2: Reference to the "Port Puant" visible on the Napoleonian cadastral plan of La-Tranche-sur-Mer produced in 1811, "Tableau d'assemblage 1811", Departmental Archives of Vendée, reference 3 P 294-1; G-3: Reference to the "Anse des terres Noires" visible on an excerpt of Claude Masse's map "Partie du Bas Poitou, générale" realised at the beginning of the 18th century, from the book of Suire, 2017; H: Legend: 1. Coring sites; 2. Breach $/$ Tidal inlet; 3. Sandy spits B and C progradations, and future progradation of the sandy spit D.

montre que la flèche sableuse est déjà bien implantée en 1924 (fig. 8). La première photographie de l'IGN prise en 1945 confirme ces observations (fig. 7C). Les dates de ces relevés cartographiques et photographiques concordent avec les datations du niveau sableux liées à la progradation de la flèche $\mathrm{D}$ (faciès $\mathrm{B}$ de BH-C, BH-A et BH-B) (fig. 3) estimée précisément entre 1908 et 1916. Par ailleurs, nous remarquons qu'un schorre était déjà implanté dans l'Anse des Rouillères sur la photographie aérienne de 1924, ce qui témoigne d'un isolement de l'océan déjà présent depuis quelques années (fig. 8). Cependant, de par leur situation à proximité de la flèche $\mathrm{C}$ et plus loin de la position actuelle de la flèche $\mathrm{D}$, les deux autres carottes lagunaires BH-D et BH-E n'ont pas enregistré cette progradation. La flèche sableuse $\mathrm{D}$ suit une progradation similaire à celle de la flèche $\mathrm{C}$ qui l'a précédée, avec une formation par dérive littorale provenant du nord-ouest vers le sud-est. Ces datations confirment donc les premiers travaux de reconstruction historique de la formation de la lagune, proposant l'initiation de sa fermeture entre 1905 et 1910. Les travaux de Bouhier questionnaient la 

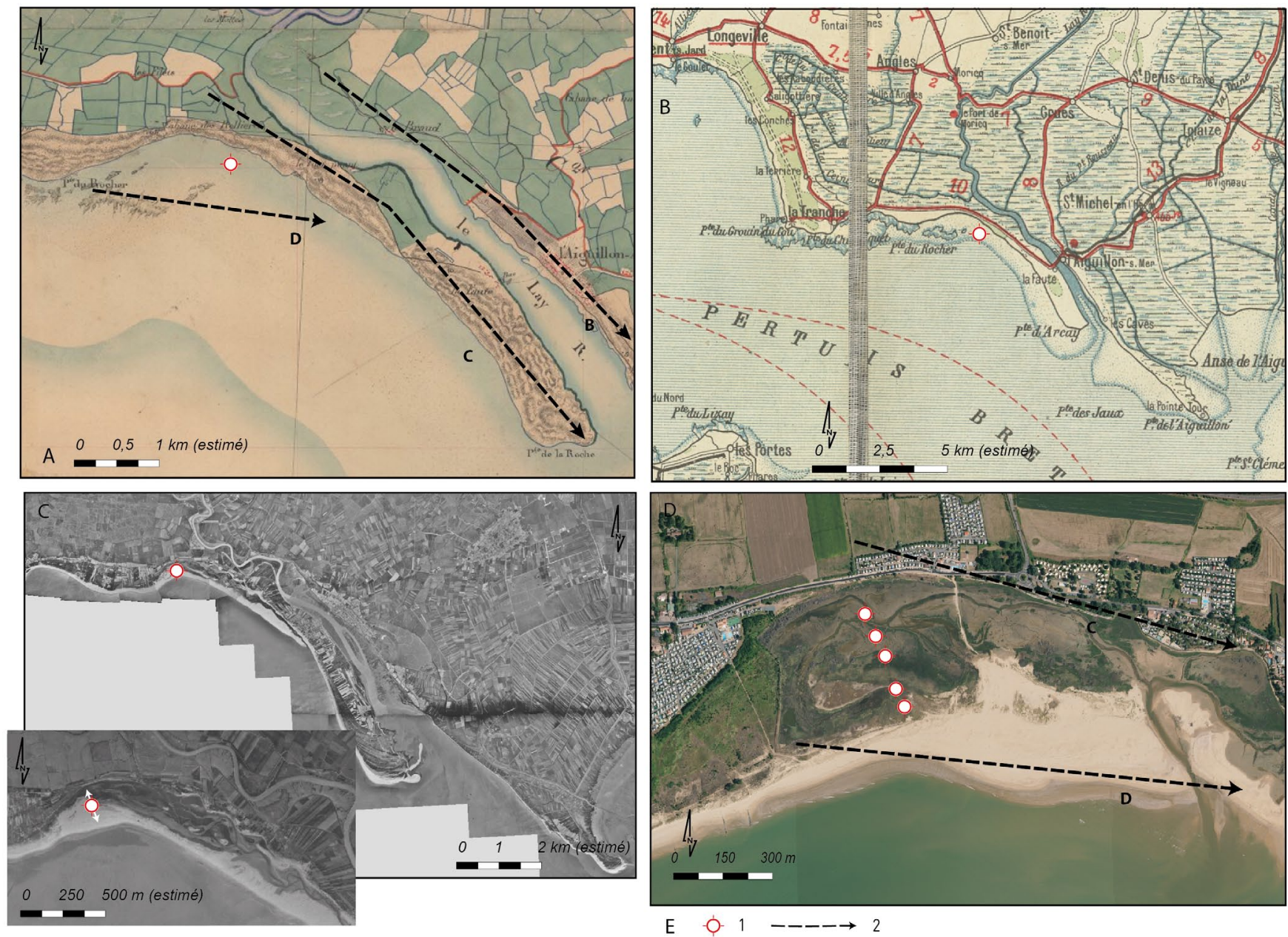

Fig. 7 - Évolution diachronique contemporaine de la lagune de la Belle-Henriette.

A : Carte de l’État-Major «FONTENAY-LE-COMTE» réalisée en 1866, issue du site internet IGN Remonter le Temps (https://remonterletemps.ign.fr/); B : Extrait de la cartographie "Carte routière pour cyclistes et automobiles de la Vendée n 13 , [1907]", éditée par A. Taride, cartographie stockée par Les bibliothèques patrimoniales de la Ville de Paris, Fonds Touring Club de France - Cote : CA 16; C : Premières photographies aériennes de l'IGN prises dans le secteur en 1945, issues du site internet IGN Remonter le Temps (https:// remonterletemps.ign.fr/) ; D : Extrait de l'IGN BD ORTHO@ de 2016 ; E : Légende : 1 . Sites de carottage ; 2. Progradations des flèches sableuses B, C et D.

\section{Fig. 7. Contemporary diachronic evolution of the Belle-Henriette lagoon.}

A: Map of the general staff "FONTENAY-LE-COMTE" created in 1866, from the website IGN Remonter le Temps (https://remonterletemps.ign.fr/); B: Excerpt from the cartography "Carte routière pour cyclistes et automobiles de la Vendée n'13, [1907]", edited by A. Taride, cartography stored in "Les bibliothèques patrimoniales de la Ville de Paris", French Touring Club funds - Classification number: CA 16; C: First aerial photographs of the IGN taken in the area in 1945, from the website IGN Remonter le Temps (https://remonterletemps.ign. fr/); D: Excerpt from the IGN BD ORTHO@ from 2016; E: Legend: 1. Coring sites; 2. Sandy spits B, C and D progradations.

création de cette flèche en lien avec le colmatage argilosilteux, en se demandant si sa progradation précédait ou succédait à ce colmatage (Bouhier, 1957). Selon nos analyses sédimentologiques, il semble que la progression de la flèche $\mathrm{D}$ précède et succède à la fois ce colmatage caractéristique argilosilteux. Ces travaux confirment également l'estimation de la création de cette flèche datant d'une " cinquantaine d'années » discutée par Fustec-Mathon et Mathon en 1960 (Fustec-Mathon et Mathon, 1960).

Enfin, après avoir été isolé de l'océan, l'environnement lagunaire actuel est composé d'une vasière à forte teneur en limons (fig. 7D), avec une végétation de schorre au plus près de la flèche sableuse $\mathrm{D}$ (BH-A et BH-B) et d'un environnement de slikke soumis au balancement des marées dans les plus faible altitudes (BH-C, $\mathrm{BH}-\mathrm{D}$ et $\mathrm{BH}-\mathrm{E})$. Les niveaux limoneux observés au sommet de ces cinq carottes, interrompus par de possibles événements extrêmes sableux, témoignent de la dynamique lagunaire actuelle (fig. 3). La combinaison des datations radio-isotopiques et des cartographies historiques anciennes permet d'estimer que la configuration sédimentologique actuelle de la lagune au niveau de l'Anse des Rouillères date de 1916.

\subsection{Traces d'occupations humaines anciennes}

Les travaux menés par Joussaume et al. (1986) apportent des marqueurs indéniables d'une occupation humaine ancienne tardive. Les plus anciennes occupations sont matérialisées par des empreintes de bovidés dans le bri, de la céramique et du matériel lithique sur une large séquence chronologique allant du Néolithique ancien vers la fin du sixième millénaire avant notre ère, jusqu'à la fin de l'âge du Bronze, soit vers env. le $2^{\mathrm{e}}$ millénaire avant notre ère. Une seconde occupation paraît quant à elle plus ponctuelle avec la découverte de structure en bois et clayonnage datés très approximativement vers 500 BC (Joussaume 


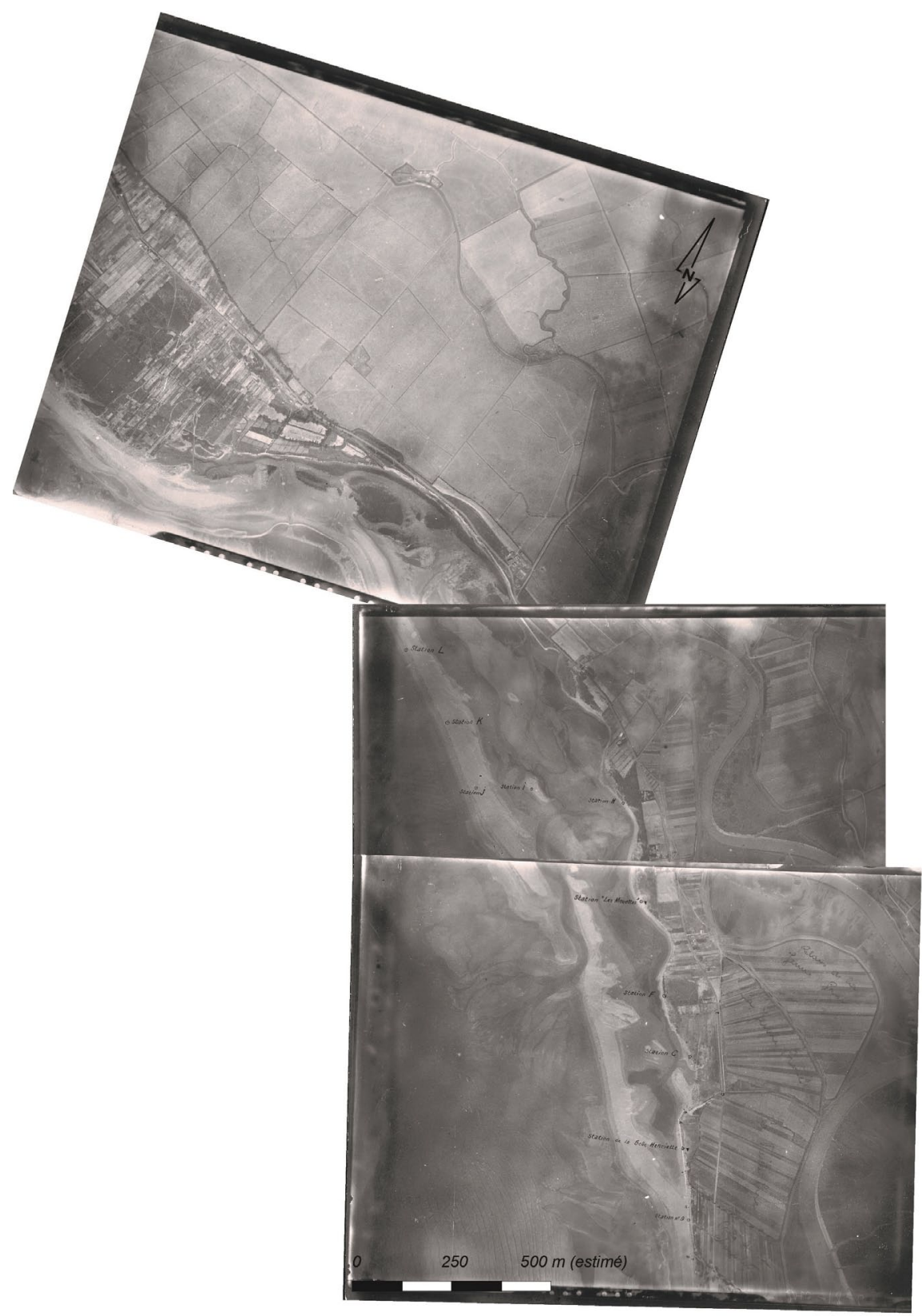

Fig. 8 - Reconstruction de la lagune de la Belle-Henriette à partir de trois photographies issues de la première campagne de photographies aériennes datant de 1923 issue des fonds de Fernand Verger.

Cette reproduction est la plus ancienne photographie aérienne de la lagune connue à ce jour.

Fig. 8 - The Reconstruction of the BelleHenriette lagoon thanks to three photographs from the first campaign of aerial photographs in 1923 from Fernand Verger's fund.

This reproduction is the oldest aerial photograph of the lagoon known to this day.

et al., 1986). En l'absence de corrélation possible entre ces anciennes occupations humaines et les aménagements côtiers empierrés que nous avons observés dans ce même secteur, nous remarquons toutefois que les pêcheries à empierrements sont toutes situées à proximité des deux points rocheux : la pointe du Grouin du Cou et la pointe du Rocher (également appelé pointe de la Grière) (fig. 4A). Ces deux ensembles rocheux sont les points d'accroche de la flèche sableuse $\mathrm{A}$ et les points de départ des flèches sableuses $C$ puis $D$, situées respectivement en arrière et en avant de la lagune de la Belle-Henriette (fig. 5B). La localisation de ces pêcheries peut s'expliquer par une grande densité de ressources halieutiques et par la proximité des matières premières pour la construction et l'entretien de ces structures empierrées. Ces positions favorables expliquent également l'occupation des Hommes à proximité. Ces rochers présentent des altitudes plus hautes (allant jusqu'à 10 à 15 m NGF selon les données issues de campagnes Lidar) que celles des marais environnants et des flèches sableuses progressant au cours des siècles sur le littoral. Ainsi, cette région a été sujette à une forte activité morphogénique durant l'Holocène. L'Île d'Yeu, localisée à moins de $100 \mathrm{~km}$ au nord-ouest de ces sites, a notamment été soumise à plusieurs phases d'augmentation de l'activité tempétueuse. Datées à 2850-2350, 5400-5370, 6650-6510, env. 7000 et entre 7670 et $7470 \mathrm{BP}$, elles concordent avec ces différentes phases d'occupations littorales (Pouzet et al., 2018)

La progradation de la flèche $\mathrm{A}$ est évaluée à une période postérieure à 540-230 BC (env. 2490-2180 BP) selon les travaux de Large (Large et al., 2017). La présence de vestiges archéologiques à proximité de ces rochers est donc expliquée par une occupation antérieure à la progradation des flèches $\mathrm{A}$ et $\mathrm{C}$, à une époque durant laquelle cet espace était encore une baie et ces deux points rocheux des îles. Si l'absence de date pour la progradation de la flèche sableuse $\mathrm{C}$ ne nous permet pas encore de répondre précisément à cette hypothèse, l'occupation humaine évaluée jusqu'à la fin de l'Âge du Bronze (Joussaume et al., 1986) surviendrait 

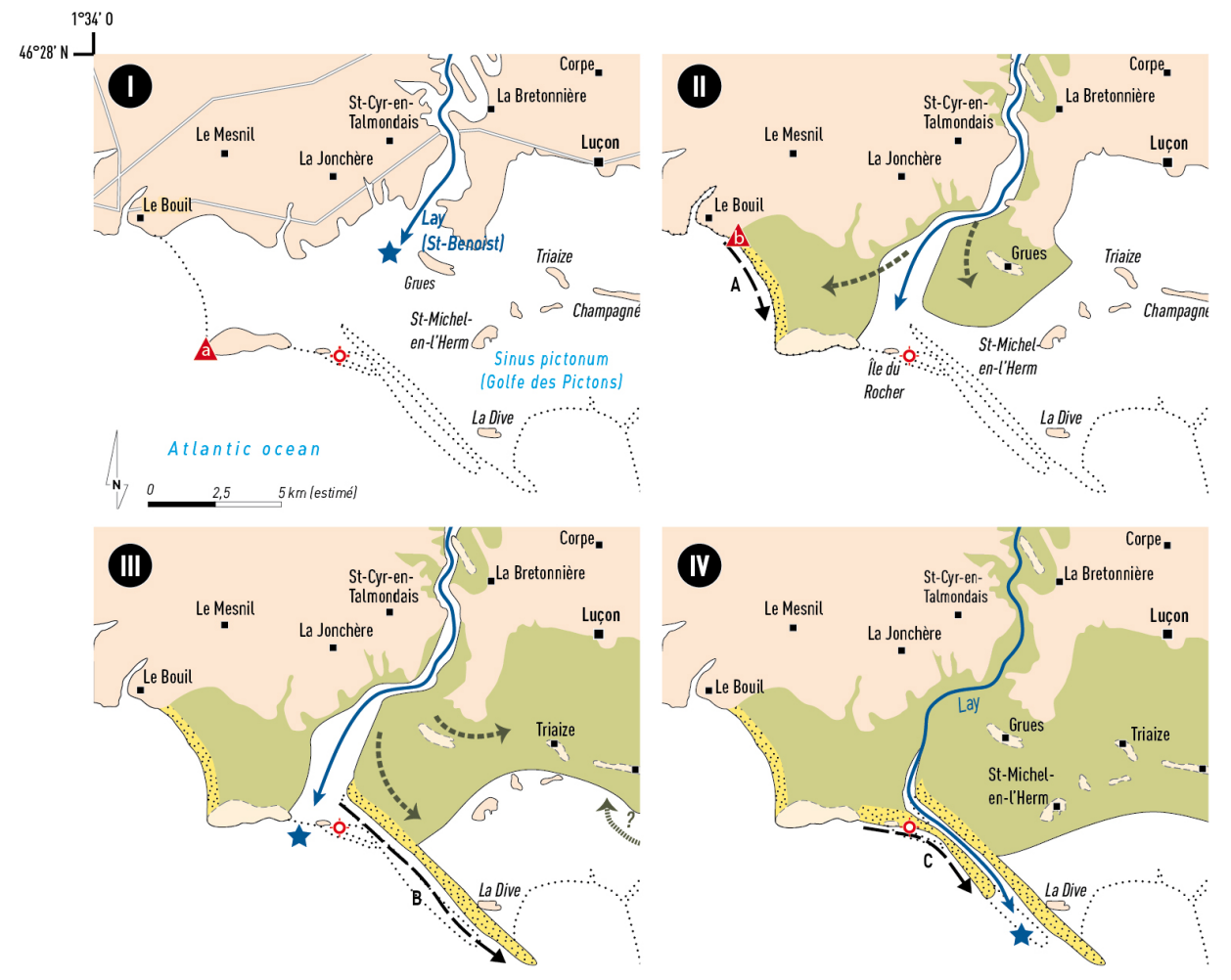

\section{Les 8 étapes de l'évolution}

(I) Golfe des Pictons

Itransgression marine liée à l'interglaciaire Holocènel

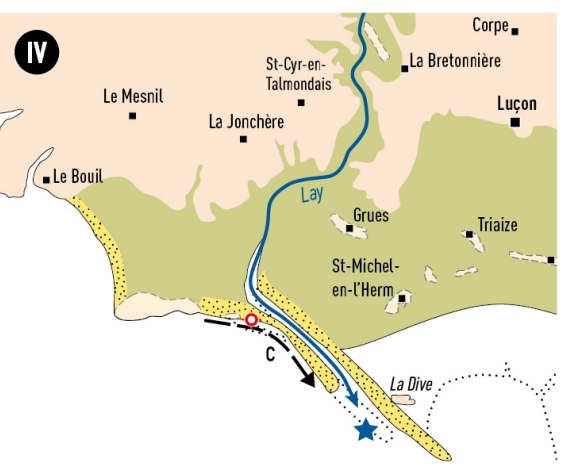

III Comblement de la rive gauche du St-Benoist suite à la progradation de la flèche $B$

(hypothèse : estimé entre la fin de ['Antiquité et le début du Moyen-Âge]
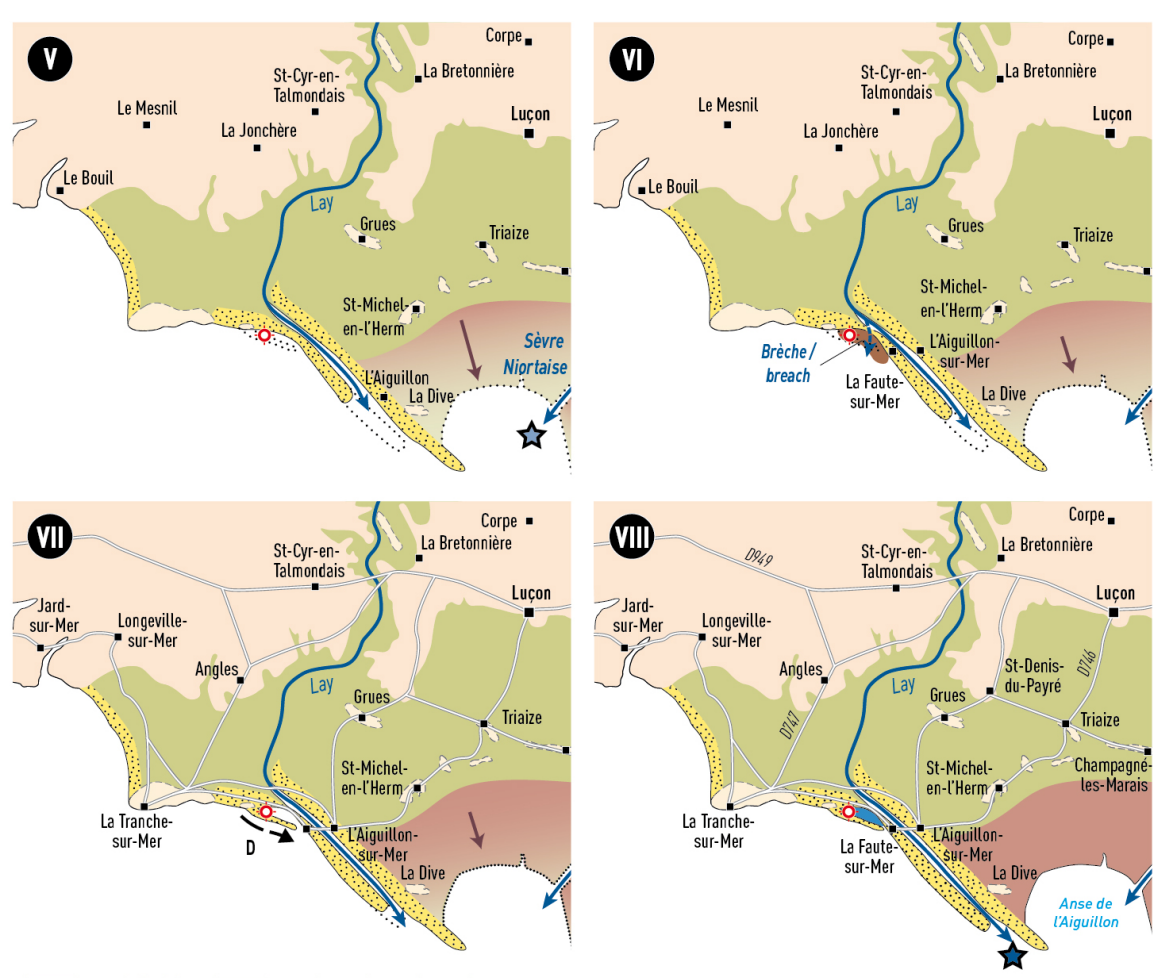

Comblement de la rive droite du St-Benoist suite à la progradation de la fleche $A$

Ihypothèse : estimé postérieure à $540-230 \mathrm{BCl}$

$$
\begin{gathered}
\text { । } \\
\text { suite } \\
\text { stimé } \\
\text { । }
\end{gathered}
$$

IV Faciès D2 (progradation flèche $C$ ) conduisant à la mise en place d'une baie maritime lfaciès D1) (hypothèse : estimé à partir de $750 \mathrm{AD}$ )

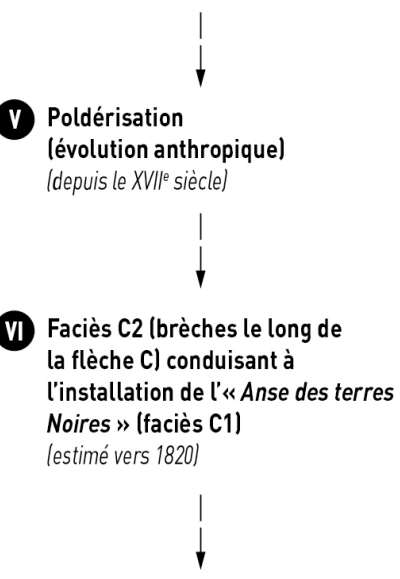

VII Faciès B (progradation flèche D) (estimé au début du XX siècle)
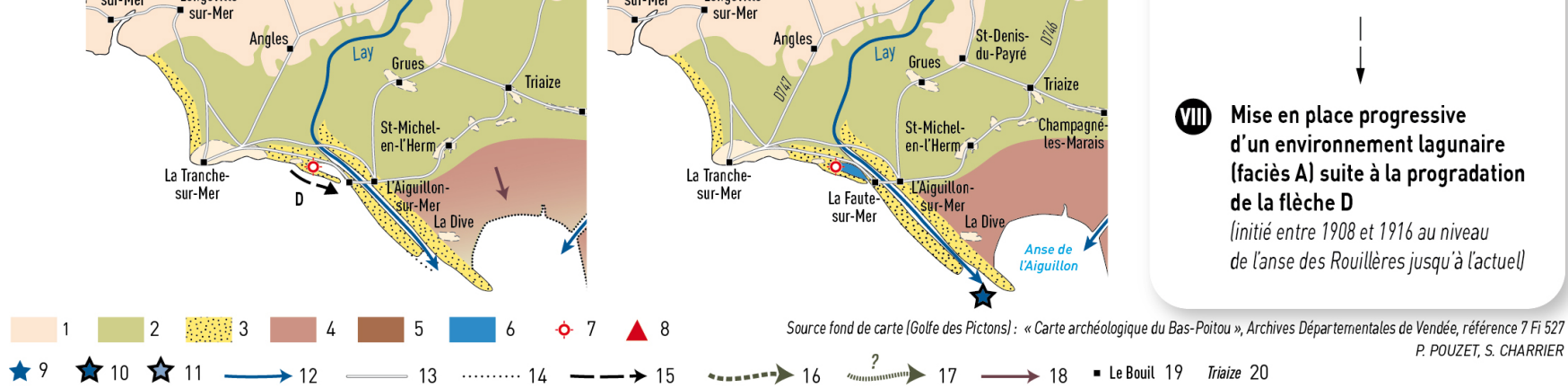

Fig. 9 - Reconstruction paléoenvironnementale du Marais poitevin occidental depuis le Golfe des Pictons.

1. Calcaire ; 2. Alluvions; 3. Sables; 4. Polders; 5. « Anse des Terres Noires »; 6. Lagune de la Belle-Henriette ; 7. Site de carottage ; 8 . Vestiges Archéologiques (a : Site de la Pointe du Grouin du Cou, occupé du $5^{\text {ème }}$ au $3^{\text {ème }}$ millénaire BC; b : Vestige daté du $2^{\text {nd }}$ Âge du Fer) ; 9. Ancienne position de l'estuaire du Lay/St-Benoist ; 10. Position actuelle de l'estuaire du Lay/St-Benoist ; 11. Estuaire actuel de la Sèvre Niortaise ; 12. Principaux fleuves ; 13. Principales routes ; 14. Trait de côte actuel ; 15. Progradation des flèches sableuses A, B, C et D ; 16. Comblement alluvionnaire du Lay/St-Benoist ; 17. Comblement alluvionnaire de la Sèvre Niortaise? ; 18. Poldérisation progressive; 19. Anciennes paroisses et communes actuelles; 20. Anciennes îles. Fig. 9 - Palaeoenvironmental reconstruction of the Western Marais Poitevin since the Gulf of Pictons.

1. Limestone; 2. Alluvium; 3. Sands; 4. Polders; 5. "Anse des Terres Noires"; 6. Belle-Henriette lagoon; 7. Coring site; 8. Archaeological remains (a: Site de la Pointe du Grouin du Cou, occupied from the 5th to the 3rd millenary BC; $b$ : Vestige dated to the 2nd Iron Age); 9. Former position of the Lay / St-Benoist estuary; 10. Current position of the Lay/St-Benoist estuary; 11. Current position of the Sèvre Niortaise estuary; 12. Main rivers; 13. Main roads; 14. Current coastline; 15. Progradation of the sandy spits A, B, C and D; 16. Alluvial filling from the Lay/St-Benoist; 17. Alluvial filling from the Sèvre Noirtaise?; 18. Progressive polderisation; 19. Former parishes and current towns; 20. Former islands. 
durant la phase de transgression marine liée à l'interglaciaire Holocène (Stéphan et Goslin, 2014; Garcia-Artola et al., 2018), et plus précisément durant la seconde phase d'affaiblissement de l'élévation du niveau marin (depuis environ 7000 ans). Selon l'analyse sédimentologique réalisée (fig. 3) et datée (fig. 2), cette occupation ancienne se situerait donc bien antérieurement à la situation de l'Anse des Terres Noires. Nous l'évaluons durant la phase allant de l'ancienne plaine antérieure à la transgression marine liée à l'interglaciaire Holocène, jusqu'à la situation du Golfe des Pictons et la progradation successive de la flèche A, qui a ensuite impulsé le comblement de l'ancienne baie. L'occupation humaine pourrait donc précisément être concordante avec la situation paléoenvironnementale du Golfe des Pictons. Cette hypothèse témoigne donc d'une occupation de l'Homme sur les anciennes îles, dans les espaces situés aux plus hautes altitudes, considérant alors pleinement la menace de l'océan. La Figure 9 synthétise l'ensemble des hypothèses proposées au sein de cette étude et esquisse la reconstruction paléoenvironnementale du Marais poitevin occidental depuis la situation du Golfe des Pictons, évaluée à l'Holocène moyen.

\section{Conclusion}

Le paysage de la partie occidentale du Marais poitevin a profondément évolué au cours des derniers millénaires. À partir de données sédimentologiques croisées avec les cartographies anciennes, nous avons identifié trois évolutions principales : (i) l'ancien Golfe des Pictons, envahi par l'océan durant la transgression marine liée à l'interglaciaire Holocène (initiée il y a 12000 ans) ; (ii) une période durant l'Antiquité et le début du Moyen Âge avec de fortes conditions morphogéniques qui ont profondément changé le paysage : le colmatage du marais par des alluvions déposées par les principaux fleuves et la progression succincte des flèches sableuses sur le littoral; (iii) la situation actuelle du marais maritime estimée après $750 \mathrm{AD}$. Dans le secteur de la Belle-Henriette, les carottages sédimentaires illustrent des variations à une échelle plus fine : (i) l'ancienne baie maritime et l'implantation de la flèche sableuse en arrière de la lagune sont identifiées dans les horizons les plus profonds (avant $1820 \mathrm{AD}$ ); (ii) l'environnement temporaire de l'Anse des Terres Noires succède à la progradation de cette flèche sableuse (entre environ 1820 et la fin du $\mathrm{XIX}^{\mathrm{e}}$ siècle); (iii) jusqu'à l'implantation de la seconde flèche sableuse, datée entre 1908 et 1916, isolant la lagune de l'océan au niveau de l'Anse des Rouillères.

Malgré ces évolutions paysagères significatives, l'occupation humaine ancienne est clairement une occupation littorale, depuis la Préhistoire jusqu'aux périodes les plus récentes. L'occupation humaine sur le long terme sans discontinuité est un fait indéniable. La présence de pêcheries en pierre s'inscrit dans une tradition de la façade atlantique d'exploitation des ressources halieutiques. Les hommes exploitent les ressources à proximité, tout en se prémunissant des aléas inhérents à une situation océanique. La seule observation de ces structures ne permet pas de fournir des indices chronologiques, c'est donc bien l'étude de ces structures et de leur environnement qu'il convient de mener. Toutes les pêcheries sont localisées à proximité des ensembles rocheux, qui présentent de plus hautes altitudes que le marais et les cordons littoraux. Par leur analyse spatiale, nous émettons l'hypothèse que l'Homme a certainement pris en compte la menace des aléas météo-marins durant ces périodes antiques.

\section{Remerciements}

Les auteurs remercient le Parc Naturel Régional (PNR) du Marais poitevin et la Ligue de Protection des Oiseaux (LPO) pour avoir fourni les autorisations de carottage, ainsi que Laurent Godet pour son aide sur la recherche de cartographies anciennes et les fonds de Fernand Verger ; Loïc Bourdy, Bastien Gruchet, Riwan Kerguillec et Martin Juigner pour leur aide durant les missions de terrain ; Simon Charrier pour la réalisation de la Figure 9 ; la plateforme PACS de l'UMR CNRS 5805 EPOC pour l'ouverture et la photographie des carottes ; et Cassandra Carnet pour la relecture de l'Anglais. Les prélèvements de terrain et analyses sédimentologiques ont été financés par la Région et la Direction Régionale de l'Environnement, de l'Aménagement et du Logement (DREAL) des Pays de la Loire dans le cadre des recherches menées au sein de l'Observatoire Régional des Risques Côtiers (OR2C - OSUNA, Axe histoire des Risques). Nous remercions également les Archives Départementales de Vendée, la Bibliothèque Nationale de France (Gallica) et Les bibliothèques patrimoniales de la Ville de Paris pour la délivrance des autorisations de diffusion des cartographies anciennes.

\section{Références}

Allard J., Bertin X., Chaumillon E., Pouget F. (2008) - Sand spit rhythmic development: A potential record of wave climate variations? Arçay Spit, western coast of France. Marine Geology, 253 (3-4), 107-131.

DOI : 10.1016/j.margeo.2008.05.009

Billard C., Bernard V. (2016) - Pêcheries de Normandie. Archéologie et histoire des pêcheries littorales du département de la Manche. Presses Universitaires de Rennes, $720 \mathrm{p}$.

Blott S.J., Pye K. (2001) - Gradistat: a grain size distribution and statistics package for the analysis of unconsolidated sediments. Earth Surface Process and Landforms, 26, 1237-1248.

DOI : $10.1002 /$ esp. 261

Bouhier A. (1957) - Aspects morphologiques de la partie occidentale du Marais Poitevin. Norois, 14, 175-207.

DOI : $10.3406 /$ noroi.1957.1181

Chapuis O. (2007) - Cartes des côtes de France; Histoire de la cartographie marine et terrestre du littoral. Glénat, $415 \mathrm{p}$.

Chaumillon E., Weber N. (2006) - Spatial variability of modern incised valleys on the French Atlantic coast: comparison between the Charente (Pertuis d'Antioche) and the Lay-Sèvre (Pertuis Breton) incised-valleys. In R.W. Dalrymple, D.A. leckie, R.W. Tillman (Eds.), Incised valleyrs in time and space, Society for Sedimentary Geology (SEPM), Tulsa, Ok, USA, 57-85.

Chaumillon E., Tessier B., Weber N., Tesson M., Bertin X. (2004) Buried sandbodies within present-day estuaries (Atlantic coast of France) revealed by very high resolution seismic surveys. Marine Geology, 221 (3-4), 189-214.

DOI: 10.1016/j.margeo.2004.07.004

Chauveau E., Chadenas C., Comentale B., Pottier P., Blanloil A., Feuillet T., Mercier D., Pourinet L., Rollo N., Tillier I., Trouillet B. (2011) - Xynthia : leçons d'une catastrophe. Cybergeo : European Journal of Geography, 24.

DOI : $10.4000 /$ cybergeo. 23763

Daire M-Y., Langouët L. (2011) - Dater les anciennes pêcheries par 
les niveaux marins, approche méthodologique et perspectives géoarchéologiques : le Bas Léon, nord Finistère, Bretagne. Norois, 220, 69-93.

DOI : $10.4000 /$ norois. 3680

Daire M-Y., Langouët L. (2010) - Les anciens pièges à poissons des côtes de Bretagne, un patrimoine eu rythme des marées. CeRAA AMARAI, Les Dossiers du Centre Régional d'Alet, AG, 165 p.

Daire M-Y., Langouët L. (2008) - Les pêcheries de Bretagne, archéologie et histoire des pêcheries d'estran. Coédition CeRAA AMARAI, nAE-2008, 144 p.

Folk R.L., Ward W.C. (1957) - Brazos River Bar: A Study in the Significance of Grain Size Parameters. Journal of Sedimentary Research, 27, 3-26.

DOI : $10.1306 / 74$ D70646-2B21-11D7-8648000102C1865D

Fustec-Mathon É., Mathon Cl.-Ch. (1960) - Notes phytoécologiques sur les formations dunaires littorales du pertuis Breton. Bulletin de la Société Botanique de France, 107, 106-110. DOI : $10.1080 / 00378941.1960 .10837978$

Garcia-Artola A., Stéphan P., Cearreta A., Kopp R., Khan N., Horton B. (2018) - Holocene sea-level database from the Atlantic coast of Europe. Quaternary Science Reviews, 196, 177-192. DOI: 10.1016/j.quascirev.2018.07.031

Gee G.W., Or D. (2002) - 2.4 Particle-Size Analysis, in: Dane J.H. Topp, C.G. (Eds.), SSSA Book Series. Soil Science Society of America, San Diego, 255-293.

DOI : $10.2136 /$ sssabookser5.4.c12

Godet L., Pourinet L., Joyeux E., Verger F. (2015) - Dynamique spatiale et usage des schorres de l'Anse de l'Aiguillon de 1705 à nos jours. Enjeux de conservation d'un patrimoine naturel littoral marin. Cybergeo : European Journal of Geography, 713.

\section{DOI : $10.4000 /$ cybergeo. 26774}

Godet L., Thomas A. (2013) - Three centuries of land cover changes in the largest French Atlantic wetland provide new insights for wetland conservation. Applied Geography, 42, 133-139.

DOI : 10.1016/j.apgeog.2013.05.011

Gruet Y., Borvon A. (2022) - Les écluses à poissons en LoireAtlantique. In : Dieulefet G. (coord.) rapport annuel du PCR La Baie de Bourgneuf dans l'économie maritime Atlantique : îles, ports et navigations, Ministère de la Culture, Direction des patrimoines, Service Régional de l'Archéologie, Département des Recherches Archéologiques Subaquatiques et Sous-marines, Université de Nantes.

Jambu P., Nijs R. (1966) - Contribution à l'étude des sols de la partie orientale du Marais Poitevin (Marais mouillé). Norois, 52, 565-593. DOI : $10.3406 /$ noroi.1966.7295

Joussaume R., Boiral M., Ters M., Denefle M., Poulain T., Délibrias G. (1986) - Sites préhistoriques submergés à la Tranche-sur-Mer (Vendée) : Etude archéologique. Bulletin de la Société préhistorique française, 83, 423-435.

DOI : 10.3406/bspf.1986.8719

Kerguillec R., Audère M., Baltzer A., Debaine F., Fattal P., Juigner M., Launeau P., Le Mauff B., Luquet F., Maanan M., Pouzet P., Robin M., Rollo N. (2019) - Monitoring and management of coastal hazards: Creation of a regional observatory of coastal erosion and storm surges in the pays de la Loire region (Atlantic coast, France). Ocean Coastal Management, 181, 104904.

DOI : 10.1016/i.ocecoaman.2019.104904
Kilmer V., Alexander L. (1949) - Methods of making mechanical analyses of soils. Soil Science, 68, 15-24.

DOI : $10.1097 / 00010694-194907000-00003$

Langouët L., Daire M-Y., Gruet Y. (2010) - Les pièges à poissons du littoral de Loire-Atlantique. Les Dossiers du Centre Régional d'Archéologie d'Alet, 38, 5-15.

Large JM., Bernard Y., Quesnel L. (2017) - Prospection géophysique sur la plage du Rocher à Longeville-sur-Mer (Vendée). Bulletin de l'AMARAI, 30, 23-24.

Large JM., Birocheau P., Corson S., Cousseau F., Large C., Tortuyaux JP. (2009) - Une archéologie des pêcheries d'estran : l'anse aux Moines et la pointe du Vieux Moulin au Château-d'Olonne, en Vendée. Groupe Vendéen d’Étude Préhistoriques, 45, 245.

Masson du Parc F. (2009, réed.) - Pêches et pêcheurs du domaine maritime et des îles adjacentes de Saintonge, d'Aunis et du Poitou, au XVIIIe siècle. Amirauté de Marennes, de la Rochelle et des Sables d'Olonne. Edition critique avec introduction, notes et index par Denis Lieppe des Procès-verbaux des visites faites par ordre du Roy concernant les pesches en mer en 1727 et en 1728. Réedition de 2009. Entre-deux-Mers, $440 \mathrm{p}$.

Mercier D., Creach A., Chevillot-Miot E., Pardo S. (2019) - After Xynthia on the Atlantic Coast of France, in: I. La Jeunesse, C. Larrue (Eds.): Facing Hydrometeorological Extreme Events. John Wiley et Sons, Ltd, 391-412. DOI : $10.1002 / 9781119383567 . c h 24$

Petuya G. (2019) - Les pêcheries insulaires du littoral vendéen (85) : les cas de l'île d'Yeu et de l'île de Noirmoutier. Master 2 recherche Archéologie, Sciences pour l'archéologie, de l'Université de Nantes, 2 volumes.

Poirier C., Tessier B., Chaumillon E., Bertin X., Fruergaard M., Mouazé D., Noël S., Weill P., Wöppelmann G. (2017) - Decadal changes in North Atlantic atmospheric circulation patterns recorded by sand spits since 1800CE. Geomorphology, 281, 1-12.

DOI : $10.1016 /$ i.geomorph.2016.12.028

Pouzet P., Creach A., Godet L. (2015) - Dynamique de la démographie et du bâti dans l'ouest du Marais poitevin depuis 1705. Norois, 234, 83-96.

DOI : 10.4000/norois.5589

Pouzet P., Maanan M., Piotrowska N., Baltzer A., Stéphan P., Robin M. (2018) - Chronology of Holocene storm events along the European Atlantic coast: New data from the Island of Yeu, France. Progress in Physical Geography: Earth and Environment, 42 (4), 431450.

\section{DOI : $10.1177 / 0309133318776500$}

Pouzet P., Maanan M., Schmidt S., Athimon E., Robin M. (2019) Correlating three centuries of historical and geological data for the marine deposit reconstruction of two depositional environments of the French Atlantic coast. Marine Geology, 407, 181-191.

DOI : 10.1016/j.margeo.2018.10.014

Pouzet P., Maanan M. (2020a) - Temporal approaches of historical extreme storm events based on sedimentological archives. Journal of African Earth Sciences, 162C, 103710.

DOI : $10.1016 /$ j.jafrearsci.2019.103710

Pouzet P., Maanan M. (2020b) - Climatological influences on major storm events during the last millennium along the Atlantic coast of France. Scientific Reports, 10, 12059. DOI : $10.1038 / \mathrm{s} 41598-020-69069-\mathrm{w}$ 
Reimer PJ, Bard E, Bayliss A, Beck JW, Blackwell PG, Bronk Ramsey C, Buck CE, Edwards RL, Friedrich M, Grootes PM, Guilderson TP, Haflidason H, Hajdas I, Hatté C, Heaton TJ, Hogg AG, Hughen KA, Kaiser KF, Kromer B, Manning SW, Reimer RW, Richards DA, Scott EM, Southon JR, Turney CSM, Van der Plicht J. (2013) - Selection and treatment of data for radiocarbon calibration: an update to the International Calibration (IntCal) criteria. Radiocarbon, 55 (4) : 1923 - 1945.

DOI : $10.2458 / \mathrm{azu}$ is rc. 55.16955

Renard J. (2005) - La Vendée, un demi-siècle d'observation d'un géographe. Presses Universitaires de Rennes, collection « Espaces et Territoires ", Rennes, 308vp.

Soulet Y. (1995) - Les écluses à poissons en pierres sèches de Noirmoutier. Lettre aux Amis. Les Amis de l'île de Noirmoutier, 98, $3-23$.

Suire Y. (2017) - Le Bas-Poitou vers 1700 ; cartes et plans et mémoires de Claude Masse, ingénieur du roi, Librairie Gallimard. ed. Paris, $369 \mathrm{p}$.

Stéphan P., Goslin J. (2014) - Holocene relative sea-level rise along the atlantic and English Channel coasts of france: reassessment of existing data using "sea-level index points" method. Quaternaire, 25, 295-312.

DOI : $10.4000 /$ quaternaire. 7269

Tardy P. (2000) - Raz-de-marée sur Ré. Les Rétais et les vimers, Groupement d'études charentaises, Les cahiers de la mémoire, 75.

Tisnérat-Laborde N., Paterne M., Métivier B., Arnold M., Yiou P., Blamart D., Raynauld S. (2010) - Variability of the northeast Atlantic sea surface $\triangle 14 \mathrm{C}$ and marine reservoir age and the North Atlantic Oscillation (NAO). Quaternary Sciences Reviews, 29, 26332646.

DOI : $10.1016 /$ j.quascirev.2010.06.013

Tessier M. (2004) - Les écluses à poissons du Pays-de-Retz (LoireAtlantique). Bulletin de l'AMARAI, 17, 83-88.

Verger F. (2009) - Zones humides du littoral français: Estuaires, deltas, marais et lagunes. Belin, Paris, 448p.

Welsch J. (1919) - Modification récente de la côte du Poitou. Annales de géographie, 28, 28-32.

DOI : $10.3406 /$ geo.1919.9370

Wolanski E., Brinson M. M., Cahoon D. R., et Perillo G. M. E. (2009) - Coastal wetlands are essential for our quality of life. In Perillo G.M.E., Wolanski E, Cahoon D.R., Brinson M.M. (Eds.): Coastal wetlands. An integrated ecosystem approach. Amsterdam, Oxford: Elsevier Science, 38-43.

Zoppi U. (2010) - Radiocarbon AMS Data Analysis: From Measured Isotopic Ratios to 14C Concentrations. Radiocarbon, 52, 165-170. DOI : $10.1017 /$ S0033822200045112

\section{Abridged English Version}

In the current context of climate change and sea-level rise, analysing past landscape evolution of vulnerable coastlines is essential. This allows us to understand ancient human occupation of societies faced with environmental mutations over the last centuries and/or millenaries. This study focuses on a very sensitive environment which was recently impacted by flooding during the storm Xynthia of February 2010. The Belle-Henriette lagoon is a coastal lagoon located between the towns of La-Faute-sur-Mer and La-Tranche-sur-Mer in the western border of the Marais Poitevin (fig. 1). According to Bouhier's works (1975), it was formed approximately a hundred years ago. The coastal wetlands act as buffer zones for the backshore areas. However, their vulnerability remains substantial. The Xynthia event struck the area severely, creating numerous breaches in the sandy spits and in dikes, flooding populated areas located behind.

To understand the crossed evolution of the environment and the adaptation of coastal societies, dated sedimentological archives permit to rebuild precisely the Belle-Henriette lagoon development and the Marais Poitevin western border infilling over the last millenaries (fig. 2-3). These palaeoenvironmental hypotheses are crossed with a landscape reconstruction based on ancient cartographic documents (fig. 5-8). Finally, the study of historical maps is also combined with the analysis of archeological remains so as to qualitatively reconstruct coastal societies' spatial mutations (fig. 4). By coupling sedimentology with historical maps, as well as with geo-archeological analyses, we try to understand how people adapted to their environment during the last millenaries. The cross analysis of palaeoenvironmental and paleosocietal evolutions in this vulnerable area gives us some keys to understanding these coevolutions.

The landscape of the western part of the Marais Poitevin has deeply evolved during the last millenaries (fig. 9). Bycrossing the sedimentological data with ancient cartographies we identified three main evolutions: (i) the former Gulf of Pictons, flooded during the marine transgression linked to Holocene interglacial (started 12000 years BP); (ii) a period during the Antiquity and the early-Middle Ages with high morphogenic dynamics that deeply changed the landscape: alluvium deposited by the main rivers contributed to the marsh infilling with the rapid progression of the sandy spits on the coast; (iii) the current situation in the Marais Poitevin estimated since post-750 AD. In the Belle-Henriette area, sediment coring illustrates the variation at a more precise scale: (i) the former marine bay and the establishment of the sandy spit behind the lagoon are identified in the deepest horizons (before 1820 AD); (ii) the temporary environment of the "Anse des Terres Noires" succeeds to this sandy spit's progradation (from nearly 1820 to the late-19 $9^{\text {th }}$ century); (iii) until the second sandy spit's establishment, which was dated between 1908 and 1916, thus isolating the lagoon from the ocean at the Anse des Rouillères location.

Despite these significant landscape evolutions, ancient human occupation is clearly defined as a coastal occupation, from prehistoric times until more recent periods. Long-term human occupation without any discontinuity is undeniable. The presence of stone fisheries is part of a tradition of exploiting halieutic resources on the Atlantic coast. People exploit resources close by while protecting themselves from the hazards that inherently happen in coastal areas. Observing these structures does not permit to find chronological clues in itself; studying these structures and environments is what needs to be done. All the fisheries are located next to rocky areas, which are higher than the marsh and the dune barriers. We assume that people considered the threat of marine and meteorological hazards during these ancient times. 